\title{
Broad kinase inhibition mitigates early neuronal dysfunction and cognitive deficits in
} tauopathy

4 Authors: Shon A. Koren ${ }^{1}$, Matthew J. Hamm ${ }^{1}$, Ryan Cloyd ${ }^{2}$, Sarah N. Fontaine ${ }^{2}$, Emad Chishti ${ }^{2}$, 5 Chiara Lanzillotta ${ }^{3}$, Jennifer Rodriguez-Rivera ${ }^{2}$, Alexandria Ingram ${ }^{2}$, Michelle Bell ${ }^{2}$, Sara M. 6 Galvis-Escobar ${ }^{1}$, Nicholas Zulia ${ }^{1}$, Fabio Di Domenico ${ }^{3}$, Duc Duong, Nicholas T. Seyfried ${ }^{4}$, 7 David Powell ${ }^{5}$, Moriel Vandsburger ${ }^{6}$, Tal Frolinger ${ }^{7}$, Anika M.S. Hartz ${ }^{2}$, John Koren $3^{\text {rd, }}{ }^{\text {, }}$ 8 Jeffrey M. Axten ${ }^{8}$, Nicholas J. Laping ${ }^{8}$, Jose F. Abisambra ${ }^{1, *}$

Affiliations:

${ }^{1}$ Department of Neuroscience \& Center for Translational Research in Neurodegenerative Disease, University of Florida, Gainesville, FL, USA.

${ }^{2}$ Sanders Brown Center on Aging \& Department of Physiology, University of Kentucky, Lexington, KY, 13 USA.

$14{ }^{3}$ Department of Biochemical Sciences, Sapienza University of Rome, Rome, Italy.

${ }^{4}$ Department of Biochemistry and Emory Integrated Proteomics Core, Emory University School of Medicine, Atlanta, GA, USA.

${ }^{5}$ Magnetic Resonance Imaging and Spectroscopy Center, University of Kentucky, Lexington, KY, USA.

${ }^{6}$ Department of Bioengineering, University of California, Berkeley, CA, USA.

${ }^{7}$ Icahn School of Medicine at Mount Sinai, New York, NY, USA.

${ }^{8}$ GlaxoSmithKline, Research and Development, Collegeville, PA, USA.

*To whom correspondence should be addressed: j.abisambra@ufl.edu

One Sentence Summary: Multi-target kinase inhibition rescues cognitive function in early stage tauopathy mice and reverses proteomic shifts common to Alzheimer's disease in humans.

Abstract: Tauopathies are a group of more than twenty known disorders that involve progressive neurodegeneration, cognitive decline, and pathological tau accumulation. Current therapeutic strategies provide only limited, late-stage symptomatic treatment. This is partly due to lack of understanding of the molecular mechanisms linking tau and cellular dysfunction, especially during the early stages of disease progression. In this study, we treated early stage tau transgenic mice with a multi-target kinase inhibitor to identify novel substrates that contribute to cognitive impairment and exhibit therapeutic potential. Drug treatment significantly ameliorated brain atrophy and cognitive function as determined by behavioral testing and a sensitive imaging technique called manganese-enhanced magnetic resonance imaging (MEMRI) with quantitative R1 mapping. Surprisingly, these benefits occurred despite unchanged hyperphosphorylated tau levels. To elucidate the mechanism behind these improved cognitive outcomes, we performed quantitative proteomics to determine the altered protein network during this early stage in tauopathy and compare this model with the human $\mathrm{AD}$ proteome. We identified a cluster of preserved pathways shared with human tauopathy with striking potential for broad multi-target kinase intervention. We further report high confidence candidate proteins as novel therapeutically relevant targets for the treatment of tauopathy. 


\section{Introduction}

Tauopathies, the most common of which is Alzheimer's disease (AD), are a group of

43 neurological disorders defined by the neuropathological accumulation of tau protein that present

44 progressive cognitive dysfunction and brain atrophy. No cure for tauopathies exists, and current

45 treatment strategies are palliative $(1,2)$. Several clinical trials have targeted toxic tau species

46 through immunotherapies or by inhibiting tau post-translational modifications and fibrillization

47 with mixed results (1-4). However, the disease etiology of tauopathies, AD in particular, are

48 almost certainly multifactorial. A promising strategy to mitigate the complex nature of

49 tauopathies, similar to established approaches for other chronic, progressive diseases, encompass

50 multi-target or combinatorial treatments $(3,5)$. These strategies are limited by requiring a

51 thorough understanding of the cellular perturbations that underly the disease.

52 Modern systems biology approaches provide unparalleled power in investigating the

53 cellular alterations in disease. These tools highlight promising candidate targets for novel

54 therapeutics while elucidating the pathophysiology of the disease. Recent studies focused on the

55 molecular network changes in $\mathrm{AD}$ suggest concurrent changes in energy metabolism, immune

56 response, synapse activity, cytoskeletal stability, and RNA metabolism pathways present early in

57 disease progression (6-8). These reports validate decades of research utilizing in vitro and in

58 vivo disease models. Using these tools, the pathways most closely associated with early cognitive

59 decline and neurodegeneration can be determined and used to assess novel therapeutics.

60 Importantly, these resources have not yet been applied to establish the efficacy of multi-target

61 drugs in mitigating cognitive decline in animal models of tauopathy.

Aberrant phosphorylation and kinase signaling is a hallmark of $\mathrm{AD}$ and other tauopathies

$63(8,9)$. Here, we report the use of a multi-target kinase inhibitor, GSK2606414 or 414, to treat tau

64 transgenic mice in a proof-of-concept study to evaluate whether such strategies can mitigate the 
65 early negative outcomes of tauopathy. This compound potently inhibits multiple tyrosine and

66 serine/threonine kinases at low micromolar concentrations (10) and ameliorates phenotypes in a

67 variety of neurological disorder and neurodegenerative models (11-16). The targets of 414

68 include kinases involved in tauopathy pathogenesis, including protein kinase R-like endoplasmic

69 reticulum kinase, or PERK (10-12), the MAPK cascade (10), receptor-interacting

70 serine/threonine-protein kinase 1, or RIPK1 (17), and KIT (18). We show that brain atrophy and

71 phenotypes of cognitive dysfunction evident in this mouse model of tauopathy are substantially

72 reduced by treatment with 414. Using manganese-enhanced MRI (MEMRI) with parametric

73 mapping, we show that multi-target kinase inhibition rescues deficits in hippocampal calcium

74 activity. The rescue of tauopathy occurs in the absence of changes to toxic, hyper-

75 phosphorylated tau levels and without inhibition of PERK, the target canonically associated with

76 the neuroprotective benefits of $414(10,11)$. We use quantitative proteomics to investigate both

77 the cellular alterations and the networks responsive to multi-target kinase inhibition associated

78 with cognitive rescue in this early stage tauopathic mouse model. Finally, we identify novel

79 candidate proteins that are rescued by drug treatment and are consistent between the tauopathy

80 mouse and human proteome. These analyses identify novel candidate targets for therapeutic

81 intervention in future studies.

\section{Results}

83 Tau transgenic mice exhibit common neurodegenerative features ameliorated by multi-target

84 kinase inhibition

85 To investigate the pathways responsible for early cognitive impairment in tauopathy and

86 their amenability to treatment with a multi-target kinase inhibitor, we used the rTg4510 tau

87 transgenic mouse model of frontotemporal lobar degeneration (FTLD). The rTg4510 (Tg) mice

88 exhibit well-characterized disease progression driven by the overexpression of P301L human tau 
89 primarily in forebrain neurons (19-21). Neurofibrillary tau pathology appears as early as two

90 months, with progressively worsening cognitive impairment detectable as early as two and a half

91 months. Additionally, significant brain atrophy is detectable in this model by 5 months of age

92 (Fig. 1A).

We treated four-month-old $\mathrm{Tg}$ and non-transgenic control (NTg) mice with the multitarget kinase inhibitor GSK2606414 (414) for 30 days. Four-month-old Tg mice display significant tau burden, but they do not present with severe cognitive impairment and therefore provide a therapeutic window amenable to treatment strategies. T2-weighted MRI (Fig. 1B-F)

97 revealed that $\mathrm{Tg}$ mice treated with vehicle $(\mathrm{Tg}+\mathrm{Veh})$ exhibited an approximately $20 \%$ reduction

98 in total brain volume compared with non-transgenic control mice treated with either vehicle

$99(\mathrm{NTg}+\mathrm{Veh}, * * \mathrm{p}=0.0063)$ or $414(\mathrm{NTg}+414, * * \mathrm{p}=0.0012)$. Surprisingly, tau transgenic mice treated with $414(\mathrm{Tg}+414)$ exhibited only a $10 \%$ reduction in total brain volume that lacked statistical significance from NTg + Veh control animals (ns, $\mathrm{p}>0.15)$. homeostasis detected by MEMRI with R1 mapping as early as 3mo of age which continues as

104 the mice age (22). Here, we corroborated those findings, identifying altered $\Delta \mathrm{R} 1 \mathrm{in} 5 \mathrm{mo} \mathrm{Tg}$ mice

105 (Fig. 2A-D). The strongest differences in $\Delta \mathrm{R} 1$ observed were found in hippocampal regions such as the dentate gyrus (DG, Fig. 2E) and Cornu ammonis area 1 (CA1, Fig. 2F). In both regions,

107 our experimental $\mathrm{Tg}+$ Veh mice exhibited significantly decreased $\Delta \mathrm{R} 1$ when compared to the 108 control NTg + Veh mice $(\mathrm{DG}, * * * \mathrm{p}=0.0003 ; \mathrm{CA} 1, * \mathrm{p}=0.0226) . \mathrm{Tg}+414$ treated mice 109 displayed no apparent impairment in calcium activity measured by MEMRI in both regions 110 relative to NTg + Veh mice (DG and CA1, ns) and displayed significant improvement in calcium 111 activity relative to the $\mathrm{Tg}+$ Veh animals $(\mathrm{DG}, * * \mathrm{p}=0.0019 ; \mathrm{CA} 1, * \mathrm{p}=0.0211)$. These findings 
112 demonstrate that, in tandem with the changes in total brain volume, treatment with the multi-

113 target kinase inhibitor 414 rescued hippocampal activity deficits in this model.

115 evaluated the effects of 414 in two behavioral paradigms: open field as a measure of anxiety-like

116 phenotype, and Y-maze as a measure of cognition. The open field behavioral paradigm

117 demonstrated a Tg phenotype wherein $\mathrm{Tg}+$ Veh mice spent more time in the field periphery than

118 either NTg group (NTg + Veh: **p =0.0012; NTg $+414: * * * p=0.0001)$, suggesting a

119 transgene-dependent anxiety-like behavior (Fig. 2G). Tg mice treated with 414 spent

120 significantly less time in the periphery than Veh treated $\operatorname{Tg}$ mice $\left({ }^{*} \mathrm{p}=0.0260\right)$ and were

121 statistically indistinguishable from either NTg treatment group (ns, $\mathrm{p}>0.15$ ). In the Y-maze, Tg

$122+$ Veh mice exhibited decreased novel arm entries $\left({ }^{*} \mathrm{p}=0.0365\right)$ compared to NTg control mice.

123 Treatment of Tg animals with 414, though statistically indistinguishable from the NTg groups

$124(\mathrm{~ns}, \mathrm{p}>0.15)$, only partially rescued the number of entries $(\mathrm{ns}, \mathrm{p}=0.1095)$ compared to vehicle-

125 treated $\mathrm{Tg}$ mice (Fig. 2H). Total time spent in the novel arm indicated $\mathrm{Tg}+\mathrm{Veh}$ mice had

126 reduced performance compared to $\mathrm{NTg}+$ Veh mice $\left({ }^{*} \mathrm{p}=0.0433\right)$. Again, the $\mathrm{Tg}+414$

127 treatment group was statistically indistinguishable from either NTg group (ns, $\mathrm{p}>0.15)$ but

128 displayed an incomplete rescue of this phenotype as compared to $\mathrm{Tg}+$ Veh mice $(\mathrm{ns}, \mathrm{p}=0.0882)$

129 (Supplemental Fig. 1). Consistent with our MEMRI and brain volume data, these data

130 demonstrated that treatment with 414 mitigates the cognitive deficits of early-stage tauopathy in

131 this model.

132 GSK2606414 rescues functional deficits without altering tau hyper-phosphorylation

133 A previous study reported that GSK2606414 reduced tau pathology by preventing PERK

134 activation and consequent enhancement of GSK3 $\beta$ activity (11). GSK3 $\beta$ phosphorylates tau at 
135 sites that are associated with tauopathy progression (24). To test whether decreased tau

136 phosphorylation was responsible for the cognitive rescue in $\mathrm{Tg}$ mice, we measured the levels of

137 disease-associated, hyper-phosphorylated tau at the S396/S404 (PHF1) and total tau levels (Fig.

138 3A). 414 did not modify the levels of PHF1, total tau, or the relative phosphorylation at the 139 PHF1 epitope (Fig. 3B-D).

140 Functional deficits of early stage tau transgenic mice do not depend on PERK/UPR activation

GSK2606414 was developed as a first-in-class inhibitor of PERK (10). PERK, an ER-

142 associated kinase, functions as part of the unfolded protein response (UPR) by attenuating global

143 translation and dampening the level of incoming nascent proteins in the ER under conditions of

144 ER stress (25). However, long term PERK activation leads to sustained suppression of

145 translation and activation of pro-apoptotic signaling cascades. Previous studies have used 414 to

146 inhibit PERK to prevent neurodegeneration by limiting the deleterious chronic activation of the

$147 \operatorname{UPR}(11,12,25)$. We evaluated whether this PERK activity was responsible for

148 neurodegeneration in early stage Tg mice. Consistent with previous reports $(11,26,27)$, PERK

149 activation (as measured by the phosphorylation of T980) was minimally detected by

150 immunofluorescent stains of the hippocampus and cortex of 5mo Tg mice (Fig. 3G-H). At 8mo,

151 Tg mice had over one hundred-fold greater levels of phosphorylated PERK (Fig. 3I-J) which was

152 inhibited by 414 treatment as previously reported (Fig. 3K) (11). Quantitative real-time PCR

153 gene card arrays verified our immunofluorescence results by demonstrating that expression

154 levels of genes downstream of PERK were largely unchanged in the 5mo Tg animals compared

155 to NTg controls and were unmodified by 414 (Fig. 3L). We measured other mediators of the

156 UPR distinct from the PERK such as ATF6, IRE1, and other eIF2 $\alpha$ kinases. Only the

157 transcriptional regulator ATF6 exhibited a significant change in transcript levels in Tg mice, with 
158 a reduction of ATF6 mRNA levels in Tg + Veh mice and 414 treated mice (Supplemental Fig.

159 2). PERK reportedly switches preferential substrate phosphorylation in tauopathies between

160 eIF2 $\alpha$ and NRF2, a transcription that promotes expression of redox response proteins (28). 414

161 treatment did not alter NRF2 nuclear translocation in Tg model mice (Fig. 3M-N). Considering

162 the off-target effects of kinase inhibitors, and those targeted by 414 beyond PERK at nanomolar

163 and low micromolar concentrations (Table S1), we reasoned that the neuroprotective effect of

164414 is likely due to a multi-target response distinct from inhibiting PERK kinase activity.

165 Quantitative hippocampal proteomics reveals pathways responsible for cognitive decline in early

166 stage tauopathy mice

167 To assess the effects of a compound impacting multiple cellular pathways

168 simultaneously, we turned to quantitative proteomics to identify proteins and pathways impacted

169 by transgenicity and our test agent, 414. Despite the prevalence of the rTg4510 model in the

170 study of neurodegeneration, no quantitative analysis of the brain proteome in these mice has

171 been reported. To this end, we evaluated the hippocampal proteome of NTg and Tg mice treated

172 with either Veh or 414 ( $\mathrm{n}=4$ per group, all female) using a multiplexed tandem mass tagging

173 (TMT) approach (Fig. 4A). Following protein quantification, batch normalization, and statistical

174 analyses, 337 proteins were identified as significantly altered across all group comparisons (FDR

175 adjusted $\mathrm{p}$ value $<0.05$, Fig. 4B). First, we identified the impact of 414 treatment on proteins in

176 NTg animals (termed: "Drug Effect") and removed these proteins from further analysis. Next,

177 we determined which proteins were altered due to $\mathrm{Tg}$ genotype (termed: "Tg Effect") by

178 comparing NTg + Veh vs Tg + Veh samples and specifically excluded any genes known to be

179 dysregulated due to transgene insertion in this model $(29,30)$. We then identified the proteins

180 that were rescued by 414 treatment in the Tg mice: proteins in the "Tg Effect" group that were 
181 restored to statistically normal levels in $\mathrm{Tg}$ mice (no significant difference from $\mathrm{NTg}+\mathrm{Veh}$

182 mice) following treatment with 414 (termed: "Drug Rescued"). These groupings are summarized

183 in (Fig. 4C) and representative proteins belonging to each group are shown in (Fig. 4D).

184 Interestingly, $\approx 67 \%$ of the proteins altered in $\mathrm{Tg}$ mice were rescued after treatment with 414 (186

185 out of 276). Importantly and as expected, the human MAPT P301L transgene was significantly

186 upregulated in Tg mice and unaffected by 414 (Fig. 4E). Tau phosphorylation was further

187 assessed using immobilized metal affinity chromatography (IMAC) enrichment and subsequent

188 LC-MS/MS for phospho-proteomic analysis. Phospho-proteomic analysis revealed tau

189 phosphorylation at 13 phospho-peptides uniquely mapping to human MAPT P301L (FDR < 1\%).

190 Averaging the abundance of these phospho-peptides as a measure of overall tau phosphorylation

191 indicated no significant difference in tau phosphorylation in between $\mathrm{Tg} 414$ treated or untreated

192 groups (Fig. 4F). Together, these proteomic data strengthen the claim that the neuroprotective

193 benefits of 414 are independent of tau.

Next, we analyzed the pathway level changes in the 5 mo $\mathrm{Tg}$ brain proteome. To collapse

195 the altered protein into pathways, we used g.Profiler (31) to assess the changes in Reactome (32)

196 annotated pathways (FDR adjusted p value < 0.1, Table S2). Cytoscape (33) was used to cluster

197 each pathway into larger umbrella networks that were down-regulated (Fig. 4G) or up-regulated

198 (Fig. 4H) in Tg mice. The down-regulated protein network clustered into nine discrete groups,

199 namely: mTOR pathway activity, synaptic activity and long-term potentiation, reactive oxygen

200 species defense, calcium dynamics, vesicle-mediated transport and endocytosis, rho GTPase

201 activity, muscle contraction, RNA metabolism and splicing, as well as aerobic respiration and

202 mitochondrial activity. The number of up-regulated protein networks was considerably larger

203 despite having fewer significantly altered proteins compared to down-regulated proteins. Up- 
204 regulated pathways clustered into the following groups: Golgi/ER transport, ion conductance,

205 immune response kinase activity, aerobic respiration and mitochondrial function, axonal and

206 neuritic morphogenesis, neurotransmitter release, amino acid metabolism, heat shock stress

207 response, insulin signaling, rho GTPase activity, cell cycle, Wnt and Hedgehog signaling, as well

208 as gap junctions and plasma membrane.

Multi-target kinase inhibition via GSK2606414 treatment rescues proteomic shifts in tauopathy

Having identified and mapped the pathway changes in Tg mice, we sought to determine

211 the pathways that were affected by 414 that associated with cognitive rescue. To that end, we

212 repeated clustering analysis of Reactome pathways (FDR adjusted p-value $<0.1$ ) representing

213 "Drug Rescued" proteins that were either initially down-regulated (Fig. 5A) or up-regulated (Fig.

214 5B) in Tg + Veh mice and rescued back toward NTg control levels with 414 treatment. Pathway

215 groups initially down-regulated in $\mathrm{Tg}+\mathrm{Veh}$ mice and rescued up toward NTg control levels

216 included: aerobic respiration, long-term potentiation, muscle contraction, ion conductance, and

217 rho GTPase activity. Other pathways initially up-regulated in $\mathrm{Tg}+$ Veh mice and restored to

218 NTg control levels included: amino acid metabolism, aerobic respiration and mitochondrial

219 function, heat shock stress response, Golgi-ER membrane trafficking, axonal and neuritic

220 morphogenesis, apoptotic protein degradation, cell cycle, gap junction and plasma membrane,

221 immune response kinase activity, ion conductance, NMDA post-synaptic activity, Wnt and

222 Hedgehog signaling, and RNA metabolism and translation.

To validate these proteomic results, we focused on two distinct cellular processes that

224 were rescued by 414: RNA translation and mitochondrial redox defense. RNA translation was

225 assessed by a novel metabolic labeling method developed to investigate the translational

226 dysregulation of tauopathy in vivo (27). This method involved an intraperitoneal injection of 
227 puromycin, a tRNA analog, shortly before death to rapidly label nascent polypeptide chains (34).

228 Anti-puromycin antibodies were then used to probe for proteins that incorporated the puromycin

229 as a direct measure of polypeptide elongation and RNA translation. Anti-puromycin staining of

230 the CA1 region revealed significantly increased translation activity in $\mathrm{Tg}+414(* \mathrm{p}<0.05)$

231 compared to $\mathrm{Tg}+$ Veh mice (Fig. 5C-D). Secondly, since nitroxidative stress is a marker of

232 mitochondrial dysregulation, and it is implicated in the pathogenesis of AD and other tauopathies

$233(35,36)$, we measured changes in protein nitration between our animal groups. Immunoblot

234 results identified a significant increase in protein nitration (3-NT) in $\mathrm{Tg}+$ Veh animals which

235 was rescued with 414 treatment (Fig. 5E-F). Together, these results validated the pathway level

236 changes that were rescued in Tg mice by treatment with 414.

237 Early stage tauopathy exhibits common protein alterations targetable by broad kinase inhibition

Finally, we investigated how the Tg hippocampal proteome compared with human

“Tg Effect" with two independent proteomic analyses of the human AD brain to identify both

241 model-specific alterations and novel candidate proteins (Table S3). The majority of significantly

242 altered mouse proteins mapped to human homologs ( $>90 \%)$ in both human tauopathy datasets, as

243 expected. First, to account for the disparity in pathology between the tauopathy Tg mouse model

244 and $\mathrm{AD}$, we assessed the consistency of proteomic alterations between $\mathrm{Tg}$ mouse and preclinical

245 (or asymptomatic) $\mathrm{AD}$ and late-stage $\mathrm{AD}$ samples (Fig. 6A). Individuals with asymptomatic $\mathrm{AD}$

246 (Asym AD) included in this analysis exhibited minimal cognitive impairment with moderate to

247 high amyloid plaque frequency, but with low to no cortical tau pathology (Table S4) (37). Late

248 AD samples, however, had significant cognitive impairment and high degrees of amyloid and tau

249 pathology. By identifying the proteins consistent between Tg mice and Asym AD distinct from 
250 late $\mathrm{AD}$, we classified which proteins are considered early markers of $\mathrm{AD}$ - and tauopathy-

251 specific progression, respectively. We found that 15 of 20 human homologs significantly altered

252 in Asym $\mathrm{AD}$ vs healthy control brains were consistently altered in $\mathrm{Tg}$ animals (75\%;

253 hypergeometric test, $* * * * p<0.0001)$. Of these 15 , nine homolog proteins were rescued by 414

254 in Tg mice: NUCB1, SNCA, FKBP1A, PKM, YWHAZ, HSPE1, GRPEL1, NDUFB10, and

255 HOMER1. These nine proteins are implicated in other neurodegenerative diseases or cellular

256 processes known to be dysregulated early in tauopathy pathogenesis such as synaptic plasticity,

257 proteostasis, glucose metabolism, and mitochondrial function. Tg mice matched to a greater

258 extent with the late AD proteome, where 29 out of 47 significantly altered proteins were inter-

259 specially consistent $(\approx 62 \%$; hypergeometric test, $* * p<0.01)$. Of these 29 proteins, 23 homologs

260 were rescued by 414 in Tg mice: NUCB1, NTM, ATOX1, SNCA, ENO1, GJA1, FKBP1A,

261 ATP6V1E1, PKM, STMN1, VSNL1, YWHAZ, HINT1, HPCA, PEPD, ROGDI, PPP1R7,

262 PACSIN1, HSPE1, RTN1, NDUFB5, DMTN, and HOMER1. These proteins function in

263 pathways rescued by 414 in $\mathrm{Tg}$ mice, namely aerobic respiration/glucose metabolism,

264 mitochondrial function, endocytosis and trafficking, immune system activation, signaling

265 cascades. Interestingly, several proteins were found consistent across disease staging: NUCB1,

266 SNCA, FKBP1A, PKM, YWHAZ, HSPE1, HOMER1. Since each of these were rescued by 414

267 in Tg mice, they may be important candidate proteins to consider for future studies.

269 to widespread brain organ failure and may not be a specific response to any one disease (38), we

270 investigated how the $\mathrm{Tg}$ proteome matched the protein alterations in $\mathrm{AD}$ and another chronic,

271 neurodegenerative disease, Parkinson's disease (PD) (39). We applied a similar approach used

272 with asymptomatic and late stage $\mathrm{AD}$, wherein we analyzed whether proteins found altered in the 
273 Tg hippocampal proteome were consistently altered in AD or PD (Fig. 6B). Interestingly, Tg

274 animals and PD brains did not share consistently altered homologous proteins, suggesting a

275 tauopathy specific effect. Of the 19 significantly altered proteins found in AD, 12 had homologs

276 consistently changed in $\mathrm{Tg}$ animals $(\approx 63 \%$; hypergeometric test $* \mathrm{p}<0.05)$. Of these twelve

277 proteins, 10 were rescued by 414: six unique to Tg animals (GJA1, EEF1A1, IDH1, SGTB,

278 PIN1, RHEB) and four proteins that are shared across both late AD (human and $\mathrm{Tg}$ ) datasets:

279 PACSIN1, GJA1, VSNL1, and NDUFB5 (Fig. 6C). These high-confidence candidate proteins

280 associated with cognitive decline and drug-mediated rescue are. Further analyses are required to

281 address the role of these candidate genes in the

\section{Discussion}

Here, we uncover critical biological processes that contribute to neurotoxic processes

284 driving cognitive dysfunction in tauopathies using a broad kinase inhibitor, GSK2606414. In

285 doing so, we demonstrate the potential to mitigate the negative functional consequences of

286 tauopathy without altering tau hyper-phosphorylation. We treated early stage rTg4510 mice and

287 firmly establish cognitive and molecular benefits of broad kinase inhibition. With TMT

288 quantitative proteomics, we identified biological processes that are affected in transgenics and

289 rescued by the compound. Finally, we cross-reference our findings to human AD and PD brain

290 proteomic data to narrow our results to high confidence unique targets for future study. This

291 approach revealed four distinct proteins associated with cognitive decline in human tauopathy

292 with strong therapeutic potential.

GSK2606414 ameliorates molecular underpinnings of neurodegeneration including

294 neuronal loss, cognitive dysfunction, and pathological protein accumulation in a variety of

295 disease models $(11-16,18)$. These studies established that the benefits of GSK2606414 were 
conferred by inhibition of its primary target, PERK, which was upregulated in disease. While

297 PERK is active in the rTg4510 tau transgenic model, this does not occur until after 6mo $(11,26)$.

298 By treating rTg4510 mice from 4-5mo, a time point in which PERK levels not activated, our

299 study identified numerous PERK-independent pathways targeted by GSK2606414 that elicited

300 rescue from toxic outcomes in tauopathy. This has important implications for data interpretation

301 of this and previous studies. abundance between soluble and insoluble fractions (40). These studies suggest that our proteomic findings reflect alterations in expression and degradation rather than a protein shift toward insolubility. studies, we searched for novel candidate targets that were associated with rescued cognitive

312 function with GSK2606414 treatment. Overlapping the proteins consistent in both AD datasets 313 with data from transgenic mice revealed four novel, high-confidence candidates that were 314 associated with cognitive decline and drug-mediated multi-target kinase inhibition: PACSIN1, 315 GJA1, VSNL1, and NDUFB5. The neuronal protein PACSIN1, or protein kinase C and casein 316 kinase substrate in neurons protein 1, participates in endocytosis (41-43) and directly interacts 317 with tubulin to promote microtubule formation (44). There is evidence for direct tau-PACSIN1 
and represents a protein with a direct link to tauopathy that could perturb cellular trafficking and tau/microtubule stability. GJA1/connexin43, or gap junction alpha 1, functions to allow rapid

321 inter-cellular communication of small molecules such as ions and neurotransmitters by 322 establishing hemichannel gap junctions between cells (46). Many studies coupled GJA1 with the 323 pathogenesis of neurodegenerative diseases such as $\mathrm{AD}$ (47, 48), most notably finding GJA1

324 incorporated into amyloid plaques (49) and upregulated in AD models and human brain (6, 50-

325 52). VSNL1, or visinin-like protein 1, is a neuronal calcium sensor (NCS) protein highly 326 expressed in the brain. As an NCS, VSNL1 responds to alterations in calcium concentration and

327 coordinates physiological processes such as cyclic nucleotide second messenger cascades and

328 synaptic receptor recycling (53-55). While the full functional role of VSNL1 remains unclear,

329 multiple studies have implicated VSNL1 in progressing calcium and synaptic dysfunction in AD

330 (56), stroke (57), and schizophrenia (58). Biomarker studies found increased VSNL1 in CSF and

331 plasma in early $\mathrm{AD}$ which correlated with cognitive dysfunction and neuronal loss (59-62).

332 NDUFB5, or nicotinamide adenine dinucleotide dehydrogenase (ubiquinone) 1 beta subcomplex

3335 , is a nuclear-encoded mitochondrial protein which functions as part of complex 1 of the

334 electron transport chain. Many studies have shown NDUFB5 levels is altered in disease,

335 typically coupled with altered expression of other mitochondrial respiratory proteins (63-66). In

336 the context of AD, NDUFB5 has been suggested as a hub protein associated with disease 337 pathogenesis based on co-expression analyses $(67,68)$.

Taken together, our pre-clinical study contributes two major findings. First, we identify

339 discrete pathways contributing to cognitive changes in tauopathy and highlight four unique

340 proteins associated with cognitive rescue with strong therapeutic potential. Further work

341 investigating the novel targets identified in this study could discover better targets amenable to 
342 pharmacological intervention and novel involvement in tauopathy. Second, we demonstrate that

343 broad, multi-family kinase inhibition can be a useful tool to mitigate the molecular and

344 functional decline in tauopathy. Since extensively multi-targeted approaches meet safety

345 challenges in the clinic, the development of compounds that selectively target key kinase

346 cascades at the same time could greatly benefit combinatorial therapies for the treatment of

347 tauopathy. Studies combining FDA-approved selective kinase inhibitors may be beneficial

348 toward advancing possible therapeutic strategies.

\section{Materials and Methods}

\section{Study Design}

352 The objective of this study was to determine the efficacy of broadly inhibiting kinase activity for

353 the treatment of early stage tauopathy. The rationale is that kinases are implicated in the early

354 pathogenesis and progression of tauopathies, such as $\mathrm{AD}$, though our understanding of the

355 dysregulated kinase network in these diseases originates from studies isolating the effect of one

356 kinase or kinase family. Given the multi-factorial complexity of these diseases, we investigated

357 whether the considerable off-target effects of a pharmacological agent (69) could provide clinical

358 benefit rather than act to confound experiments. Therefore, we evaluated the effect of a

359 compound originally developed as a selective inhibitor for PERK (10) and used in that context in

360 a variety of disease models $(11,12,16)$, but which reportedly broadly targets kinases at low

361 micromolar concentrations $(10,17,18)$. We tested the outcomes of treating tau transgenic mice

362 with this compound across a variety of functional, cognitive, and molecular measures of

363 tauopathy. We confirmed that the neuroprotective effect of the compound is independent of

364 PERK activity at this early age, though older mice with PERK activity respond to the compound 
365 as previously reported. Lastly, we investigated the protein changes underlying the cognitive

366 dysfunction and subsequent rescue at this age in tau transgenic mice. To control for transgene-

367 specific effects in mice which may not be recapitulated tauopathy in humans, we compared these

368 results with two separate measures of the $\mathrm{AD}$ proteome using different proteomic techniques to

369 control for potential systematic biases.

370 All experiments were designed with appropriate controls and based on previous experiments to

371 determine statistical power $(22,27,70)$. Animals were separated by genotype and randomly

372 assigned to treatment groups and experimental cohorts. Mice which did not undergo full

373 treatment course, such as those removed from analysis due to body weight reduction past $80 \%$ of

374 starting weight, were not analyzed for this study.

\section{Animals}

376 All animal studies were approved by the University of Kentucky's Institutional Animal Care and

377 Use Committee (IACUC) and abided by that committee's policies on animal care and use in

378 accordance with federal guidelines. Mice were kept in standard housing on a $12 \mathrm{~h}$ light/dark cycle

379 and received food and water ad libitum. The tau transgenic (rTg4510) and parental mice were

380 maintained and genotyped as described previously $(20,27)$ and were maintained on mixed FVB

381 and 129S6 backgrounds. Mice of both sexes were used in experiments unless otherwise stated.

\section{Treatment with GSK2606414}

383 GSK2606414 (GlaxoSmithKline) was suspended in vehicle (0.5\% hydroxypropylmethyl

384 cellulose $+0.1 \%$ Tween-80 in water at $\mathrm{pH} 4.0$ ) as previously described (12). A total of 100

$385 \mathrm{mg} / \mathrm{kg}$ GSK2606414 was delivered by oral gavage once $(100 \mathrm{mg} / \mathrm{kg})$ or twice a day $(50 \mathrm{mg} / \mathrm{kg}$

386 each separated by 10-12 hours). Prior to treatment, mice were handled twice daily to acclimate 
the animals for gavage. Unless otherwise stated, four-month-old rTg4510 and non-transgenic mice were treated with GSK2606414 or vehicle for 30-33 days ranging to 36 days to accommodate for environmental habituation for behavioral and MRI assays. Daily weight records were kept to ensure accurate dosing and to monitor potential GSK2606414-mediated weight loss (12). Animals that lost more than $20 \%$ of starting body weight were excluded from the study (Supplemental Fig. 3). No group exhibited pronounced weight loss.

\section{Statistical Analysis}

394 Statistical analyses for all data apart from proteomics were performed using GraphPad Prism 8

395 (Graph Pad Software, Inc. La Jolla, CA, USA). Results are shown as the mean \pm standard error 396 or standard deviation as described in each figure. Single-variate data were analyzed using 397 unpaired Student's t-test. Multi-variate data were analyzed with one-way or two-way ANOVA 398 where appropriate, corrected for multiple comparisons with Tukey post-test analysis unless 399 otherwise stated. A value of $\mathrm{p}<0.05$ was considered statistically significant except for Reactome 400 pathway analyses which used an FDR-adjusted p value cutoff of 0.10 .

401

\section{References} Strategies for the Treatment of Tauopathies: Hopes and Challenges, Alzheimers Dement 12, 405 1051-1065 (2016). Neuropathologica Communications 7, 22 (2019).

409 3. L.-K. Huang, S.-P. Chao, C.-J. Hu, Clinical trials of new drugs for Alzheimer disease, Journal of 410 Biomedical Science 27, 18 (2020).

411 4. A. L. Boxer, A. E. Lang, M. Grossman, D. S. Knopman, B. L. Miller, L. S. Schneider, R. S. Doody, 412 A. Lees, L. I. Golbe, D. R. Williams, J.-C. Corvol, A. Ludolph, D. Burn, S. Lorenzl, I. Litvan, E. D. 
Roberson, G. U. Höglinger, M. Koestler, C. R. Jack, V. Van Deerlin, C. Randolph, I. V. Lobach, H. W. Heuer, I. Gozes, L. Parker, S. Whitaker, J. Hirman, A. J. Stewart, M. Gold, B. H. Morimoto, AL108-231 Investigators, Davunetide in patients with progressive supranuclear palsy: a randomised, double-blind, placebo-controlled phase 2/3 trial, Lancet Neurol 13, 676-685 (2014).

5. R. R. Ramsay, M. R. Popovic-Nikolic, K. Nikolic, E. Uliassi, M. L. Bolognesi, A perspective on multi-target drug discovery and design for complex diseases, Clinical and Translational Medicine 7, 3 (2018).

6. E. C. B. Johnson, E. B. Dammer, D. M. Duong, L. Ping, M. Zhou, L. Yin, L. A. Higginbotham, A. Guajardo, B. White, J. C. Troncoso, M. Thambisetty, T. J. Montine, E. B. Lee, J. Q. Trojanowski, T. G. Beach, E. M. Reiman, V. Haroutunian, M. Wang, E. Schadt, B. Zhang, D. W. Dickson, N. Ertekin-Taner, T. E. Golde, V. A. Petyuk, P. L. De Jager, D. A. Bennett, T. S. Wingo, S. Rangaraju, I. Hajjar, J. M. Shulman, J. J. Lah, A. I. Levey, N. T. Seyfried, Large-scale proteomic analysis of Alzheimer's disease brain and cerebrospinal fluid reveals early changes in energy metabolism associated with microglia and astrocyte activation, Nat. Med. 26, 769-780 (2020).

7. E. C. B. Johnson, E. B. Dammer, D. M. Duong, L. Yin, M. Thambisetty, J. C. Troncoso, J. J. Lah, A. I. Levey, N. T. Seyfried, Deep proteomic network analysis of Alzheimer's disease brain reveals alterations in RNA binding proteins and RNA splicing associated with disease, $\mathrm{Mol}$ Neurodegener 13, 52 (2018).

8. B. Bai, X. Wang, Y. Li, P.-C. Chen, K. Yu, K. K. Dey, J. M. Yarbro, X. Han, B. M. Lutz, S. Rao, Y. Jiao, J. M. Sifford, J. Han, M. Wang, H. Tan, T. I. Shaw, J.-H. Cho, S. Zhou, H. Wang, M. Niu, A. Mancieri, K. A. Messler, X. Sun, Z. Wu, V. Pagala, A. A. High, W. Bi, H. Zhang, H. Chi, V. Haroutunian, B. Zhang, T. G. Beach, G. Yu, J. Peng, Deep Multilayer Brain Proteomics Identifies Molecular Networks in Alzheimer's Disease Progression, Neuron 105, 975-991.e7 (2020).

9. M. Perluigi, E. Barone, F. Di Domenico, D. A. Butterfield, Aberrant protein phosphorylation in Alzheimer disease brain disturbs pro-survival and cell death pathways, Biochim. Biophys. Acta 1862, 1871-1882 (2016).

10. J. M. Axten, J. R. Medina, Y. Feng, A. Shu, S. P. Romeril, S. W. Grant, W. H. H. Li, D. A. Heerding, E. Minthorn, T. Mencken, C. Atkins, Q. Liu, S. Rabindran, R. Kumar, X. Hong, A. Goetz, T. Stanley, J. D. Taylor, S. D. Sigethy, G. H. Tomberlin, A. M. Hassell, K. M. Kahler, L. M. Shewchuk, R. T. Gampe, Discovery of 7-methyl-5-(1-\{[3-(trifluoromethyl)phenyl]acetyl\}-2,3dihydro-1H-indol-5-yl)-7H-pyrrolo[2,3-d]pyrimidin-4-amine (GSK2606414), a potent and selective first-in-class inhibitor of protein kinase $R$ (PKR)-like endoplasmic reticulum kinase (PERK), J. Med. Chem. 55, 7193-7207 (2012).

11. H. Radford, J. A. Moreno, N. Verity, M. Halliday, G. R. Mallucci, PERK inhibition prevents taumediated neurodegeneration in a mouse model of frontotemporal dementia, Acta Neuropathol 130, 633-642 (2015). 
12. J. A. Moreno, M. Halliday, C. Molloy, H. Radford, N. Verity, J. M. Axten, C. A. Ortori, A. E. Willis, P. M. Fischer, D. A. Barrett, G. R. Mallucci, Oral treatment targeting the unfolded protein response prevents neurodegeneration and clinical disease in prion-infected mice, Sci TransI Med 5, 206ra138 (2013).

13. C. Meng, J. Zhang, B. Dang, H. Li, H. Shen, X. Li, Z. Wang, PERK Pathway Activation Promotes Intracerebral Hemorrhage Induced Secondary Brain Injury by Inducing Neuronal Apoptosis Both in Vivo and in Vitro, Front Neurosci 12 (2018), doi:10.3389/fnins.2018.00111.

\section{7}

14. T. Vanderweyde, D. J. Apicco, K. Youmans-Kidder, P. E. A. Ash, C. Cook, E. L. da Rocha, K. Jansen-West, A. A. Frame, A. Citro, J. D. Leszyk, P. Ivanov, J. F. Abisambra, M. Steffen, H. Li, L. Petrucelli, B. Wolozin, Interaction of tau with the RNA-Binding Protein TIA1 Regulates tau Pathophysiology and Toxicity, Cell Rep 15, 1455-1466 (2016).

15. V. Sharma, H. Ounallah-Saad, D. Chakraborty, M. Hleihil, R. Sood, I. Barrera, E. Edry, S. K. Chandran, S. B. T. de Leon, H. Kaphzan, K. Rosenblum, Local Inhibition of PERK Enhances Memory and Reverses Age-Related Deterioration of Cognitive and Neuronal Properties, J. Neurosci. 38, 648-658 (2018).

16. H. L. Smith, O. J. Freeman, A. J. Butcher, S. Holmqvist, I. Humoud, T. Schätzl, D. T. Hughes, N. C. Verity, D. P. Swinden, J. Hayes, L. de Weerd, D. H. Rowitch, R. J. M. Franklin, G. R. Mallucci, Astrocyte Unfolded Protein Response Induces a Specific Reactivity State that Causes Non-CellAutonomous Neuronal Degeneration, Neuron 105, 855-866.e5 (2020).

17. D. Rojas-Rivera, T. Delvaeye, R. Roelandt, W. Nerinckx, K. Augustyns, P. Vandenabeele, M. J. M. Bertrand, When PERK inhibitors turn out to be new potent RIPK1 inhibitors: critical issues on the specificity and use of GSK2606414 and GSK2656157, Cell Death Differ 24, 1100-1110 (2017).

18. M. Mahameed, T. Wilhelm, O. Darawshi, A. Obiedat, W.-S. Tommy, C. Chintha, T. Schubert, A. Samali, E. Chevet, L. A. Eriksson, M. Huber, B. Tirosh, The unfolded protein response modulators GSK2606414 and KIRA6 are potent KIT inhibitors, Cell Death Dis 10, 300 (2019).

19. Z. Berger, H. Roder, A. Hanna, A. Carlson, V. Rangachari, M. Yue, Z. Wszolek, K. Ashe, J. Knight, D. Dickson, C. Andorfer, T. L. Rosenberry, J. Lewis, M. Hutton, C. Janus, Accumulation of Pathological Tau Species and Memory Loss in a Conditional Model of Tauopathy, J. Neurosci. 27, 3650-3662 (2007).

20. K. SantaCruz, J. Lewis, T. Spires, J. Paulson, L. Kotilinek, M. Ingelsson, A. Guimaraes, M. DeTure, M. Ramsden, E. McGowan, C. Forster, M. Yue, J. Orne, C. Janus, A. Mariash, M. Kuskowski, B. Hyman, M. Hutton, K. H. Ashe, Tau Suppression in a Neurodegenerative Mouse Model Improves Memory Function, Science 309, 476-481 (2005). 
21. R. M. Bailey, J. Howard, J. Knight, N. Sahara, D. W. Dickson, J. Lewis, Effects of the C57BL/6 strain background on tauopathy progression in the rTg4510 mouse model, Molecular Neurodegeneration 9, 8 (2014).

22. S. N. Fontaine, A. Ingram, R. A. Cloyd, S. E. Meier, E. Miller, D. Lyons, G. K. Nation, E. Mechas, B. Weiss, C. Lanzillotta, F. Di Domenico, F. Schmitt, D. K. Powell, M. Vandsburger, J. F. Abisambra, Identification of changes in neuronal function as a consequence of aging and tauopathic neurodegeneration using a novel and sensitive magnetic resonance imaging approach, Neurobiol Aging 56, 78-86 (2017).

23. F. M. LaFerla, Calcium dyshomeostasis and intracellular signalling in Alzheimer's disease, Nat. Rev. Neurosci. 3, 862-872 (2002).

24. C. Hooper, R. Killick, S. Lovestone, The GSK3 hypothesis of Alzheimer's disease, J Neurochem 104, 1433-1439 (2008). (2016).

26. J. F. Abisambra, U. K. Jinwal, L. J. Blair, J. C. O'Leary, Q. Li, S. Brady, L. Wang, C. E. Guidi, B. Zhang, B. A. Nordhues, M. Cockman, A. Suntharalingham, P. Li, Y. Jin, C. A. Atkins, C. A. Dickey, Tau accumulation activates the unfolded protein response by impairing endoplasmic reticulumassociated degradation, J. Neurosci. 33, 9498-9507 (2013). Chen, H. Zhu, E. M. Blalock, J. F. Abisambra, Tau drives translational selectivity by interacting with ribosomal proteins, Acta Neuropathol 137, 571-583 (2019).

506 28. S. B. Cullinan, D. Zhang, M. Hannink, E. Arvisais, R. J. Kaufman, J. A. Diehl, Nrf2 is a direct PERK substrate and effector of PERK-dependent cell survival, Mol. Cell. Biol. 23, 7198-7209

508 (2003).

509 29. L. O. Goodwin, E. Splinter, T. L. Davis, R. Urban, H. He, R. E. Braun, E. J. Chesler, V. Kumar, M. 510 van Min, J. Ndukum, V. M. Philip, L. G. Reinholdt, K. Svenson, J. K. White, M. Sasner, C. Lutz, S. 511 A. Murray, Large-scale discovery of mouse transgenic integration sites reveals frequent 512 structural variation and insertional mutagenesis, Genome Res. 29, 494-505 (2019).

513 30. J. Gamache, K. Benzow, C. Forster, L. Kemper, C. Hlynialuk, E. Furrow, K. H. Ashe, M. D. 514 Koob, Factors other than hTau overexpression that contribute to tauopathy-like phenotype in 515 rTg4510 mice, Nat Commun 10, 2479 (2019).

516 31. U. Raudvere, L. Kolberg, I. Kuzmin, T. Arak, P. Adler, H. Peterson, J. Vilo, g:Profiler: a web 517 server for functional enrichment analysis and conversions of gene lists (2019 update), Nucleic 518 Acids Res 47, W191-W198 (2019). 
32. B. Jassal, L. Matthews, G. Viteri, C. Gong, P. Lorente, A. Fabregat, K. Sidiropoulos, J. Cook, M. Gillespie, R. Haw, F. Loney, B. May, M. Milacic, K. Rothfels, C. Sevilla, V. Shamovsky, S. Shorser, T. Varusai, J. Weiser, G. Wu, L. Stein, H. Hermjakob, P. D’Eustachio, The reactome pathway knowledgebase, Nucleic Acids Res. 48, D498-D503 (2020).

33. P. Shannon, A. Markiel, O. Ozier, N. S. Baliga, J. T. Wang, D. Ramage, N. Amin, B. Schwikowski, T. Ideker, Cytoscape: a software environment for integrated models of biomolecular interaction networks, Genome Res. 13, 2498-2504 (2003).

34. C. A. Goodman, T. A. Hornberger, Measuring protein synthesis with SUnSET: a valid alternative to traditional techniques?, Exerc Sport Sci Rev 41, 107-115 (2013).

35. D. A. Butterfield, T. T. Reed, M. Perluigi, C. De Marco, R. Coccia, J. N. Keller, W. R. Markesbery, R. Sultana, Elevated Levels of 3-Nitrotyrosine in Brain From Subjects with Amnestic Mild Cognitive Impairment: Implications for the Role of Nitration in the Progression of Alzheimer's Disease, Brain Res 1148, 243-248 (2007).

36. D. A. Butterfield, T. Reed, R. Sultana, Roles of 3-nitrotyrosine- and 4-hydroxynonenalmodified brain proteins in the progression and pathogenesis of Alzheimer's disease, Free Radic. Res. 45, 59-72 (2011).

37. N. T. Seyfried, E. B. Dammer, V. Swarup, D. Nandakumar, D. M. Duong, L. Yin, Q. Deng, T. Nguyen, C. M. Hales, T. Wingo, J. Glass, M. Gearing, M. Thambisetty, J. C. Troncoso, D. H. Geschwind, J. J. Lah, A. I. Levey, A Multi-network Approach Identifies Protein-Specific Coexpression in Asymptomatic and Symptomatic Alzheimer's Disease, Cell Syst 4, 60-72.e4 (2017).

38. T. E. Golde, S. T. DeKosky, D. Galasko, Alzheimer's disease: The right drug, the right time, Science 362, 1250-1251 (2018).

39. L. Ping, D. M. Duong, L. Yin, M. Gearing, J. J. Lah, A. I. Levey, N. T. Seyfried, Global quantitative analysis of the human brain proteome in Alzheimer's and Parkinson's Disease, Sci Data 5, 180036 (2018).

40. M. C. Pace, G. Xu, S. Fromholt, J. Howard, K. Crosby, B. I. Giasson, J. Lewis, D. R. Borchelt, Changes in proteome solubility indicate widespread proteostatic disruption in mouse models of neurodegenerative disease, Acta Neuropathol 136, 919-938 (2018).

41. J. Modregger, B. Ritter, B. Witter, M. Paulsson, M. Plomann, All three PACSIN isoforms bind to endocytic proteins and inhibit endocytosis, J. Cell. Sci. 113 Pt 24, 4511-4521 (2000).

42. V. Anggono, K. J. Smillie, M. E. Graham, V. A. Valova, M. A. Cousin, P. J. Robinson, Syndapin I is the phosphorylation-regulated dynamin I partner in synaptic vesicle endocytosis, Nat.

Neurosci. 9, 752-760 (2006). 
552 43. A. Braun, R. Pinyol, R. Dahlhaus, D. Koch, P. Fonarev, B. D. Grant, M. M. Kessels, B. 553 Qualmann, EHD proteins associate with syndapin I and II and such interactions play a crucial 554 role in endosomal recycling, Mol. Biol. Cell 16, 3642-3658 (2005).

555 44. E.-M. S. Grimm-Günter, M. Milbrandt, B. Merkl, M. Paulsson, M. Plomann, PACSIN proteins 556 bind tubulin and promote microtubule assembly, Exp. Cell Res. 314, 1991-2003 (2008).

557 45. Y. Liu, K. Lv, Z. Li, A. C. H. Yu, J. Chen, J. Teng, PACSIN1, a Tau-interacting protein, regulates 558 axonal elongation and branching by facilitating microtubule instability, J. Biol. Chem. 287, 559 39911-39924 (2012).

560 46. D. A. Goodenough, D. L. Paul, Beyond the gap: functions of unpaired connexon channels, 561 Nat. Rev. Mol. Cell Biol. 4, 285-294 (2003).

562 47. J.-T. He, X.-Y. LI, L. Yang, X. Zhao, Astroglial connexins and cognition: memory formation or 563 deterioration?, Biosci Rep 40 (2020), doi:10.1042/BSR20193510.

564 48. L. C. Mayorquin, A. V. Rodriguez, J.-J. Sutachan, S. L. Albarracín, Connexin-Mediated 565 Functional and Metabolic Coupling Between Astrocytes and Neurons, Front Mol Neurosci 11 566 (2018), doi:10.3389/fnmol.2018.00118.

567 49. J. I. Nagy, W. Li, E. L. Hertzberg, C. A. Marotta, Elevated connexin43 immunoreactivity at 568 sites of amyloid plaques in Alzheimer's disease, Brain Res. 717, 173-178 (1996).

569 50. R. Ren, L. Zhang, M. Wang, Specific deletion connexin43 in astrocyte ameliorates cognitive 570 dysfunction in APP/PS1 mice, Life Sci. 208, 175-191 (2018).

571 51. X. Mei, P. Ezan, C. Giaume, A. Koulakoff, Astroglial connexin immunoreactivity is specifically 572 altered at $\beta$-amyloid plaques in $\beta$-amyloid precursor protein/presenilin1 mice, Neuroscience 573 171, 92-105 (2010).

574 52. Y. Kajiwara, E. Wang, M. Wang, W. C. Sin, K. J. Brennand, E. Schadt, C. C. Naus, J. Buxbaum, 575 B. Zhang, GJA1 (connexin43) is a key regulator of Alzheimer's disease pathogenesis, Acta 576 Neuropathol Commun 6, 144 (2018).

577 53. M. Brackmann, S. Schuchmann, R. Anand, K.-H. Braunewell, Neuronal Ca2+ sensor protein 578 VILIP-1 affects CGMP signalling of guanylyl cyclase B by regulating clathrin-dependent receptor 579 recycling in hippocampal neurons, J. Cell. Sci. 118, 2495-2505 (2005).

580 54. R. D. Burgoyne, Neuronal calcium sensor proteins: generating diversity in neuronal Ca2+ 581 signalling, Nat. Rev. Neurosci. 8, 182-193 (2007).

582 55. K.-H. Braunewell, A. J. Klein-Szanto, Visinin-like proteins (VSNLs): interaction partners and 583 emerging functions in signal transduction of a subfamily of neuronal Ca2+-sensor proteins, Cell 584 Tissue Res 335, 301-316 (2009). 
56. M. Groblewska, P. Muszyński, A. Wojtulewska-Supron, A. Kulczyńska-Przybik, B. Mroczko, The Role of Visinin-Like Protein-1 in the Pathophysiology of Alzheimer's Disease, J. Alzheimers Dis. 47, 17-32 (2015).

57. O. F. Laterza, V. R. Modur, D. L. Crimmins, J. V. Olander, Y. Landt, J.-M. Lee, J. H. Ladenson, Identification of novel brain biomarkers, Clin. Chem. 52, 1713-1721 (2006).

58. K. H. Braunewell, A. D. Dwary, F. Richter, K. Trappe, C. Zhao, I. Giegling, K. Schönrath, D. Rujescu, Association of VSNL1 with schizophrenia, frontal cortical function, and biological significance for its gene product as a modulator of CAMP levels and neuronal morphology, Transl Psychiatry 1, e22 (2011).

59. J.-M. Lee, K. Blennow, N. Andreasen, O. Laterza, V. Modur, J. Olander, F. Gao, M. Ohlendorf, J. H. Ladenson, The brain injury biomarker VLP-1 is increased in the cerebrospinal fluid of Alzheimer disease patients, Clin. Chem. 54, 1617-1623 (2008).

60. R. Tarawneh, G. D’Angelo, E. Macy, C. Xiong, D. Carter, N. J. Cairns, A. M. Fagan, D. Head, M. A. Mintun, J. H. Ladenson, J.-M. Lee, J. C. Morris, D. M. Holtzman, Visinin-like protein-1: diagnostic and prognostic biomarker in Alzheimer disease, Ann. Neurol. 70, 274-285 (2011).

61. R. Tarawneh, J.-M. Lee, J. H. Ladenson, J. C. Morris, D. M. Holtzman, CSF VILIP-1 predicts rates of cognitive decline in early Alzheimer disease, Neurology 78, 709-719 (2012).

62. C. M. Kirkwood, M. L. MacDonald, T. A. Schempf, A. V. Vatsavayi, M. D. Ikonomovic, J. L. Koppel, Y. Ding, M. Sun, J. K. Kofler, O. L. Lopez, N. A. Yates, R. A. Sweet, Altered Levels of Visinin-Like Protein 1 Correspond to Regional Neuronal Loss in Alzheimer Disease and Frontotemporal Lobar Degeneration, J. Neuropathol. Exp. Neurol. 75, 175-182 (2016).

63. P. M. Keeney, J. Xie, R. A. Capaldi, J. P. Bennett, Parkinson's disease brain mitochondrial complex I has oxidatively damaged subunits and is functionally impaired and misassembled, $J$. Neurosci. 26, 5256-5264 (2006).

64. P. Andrés-Benito, E. Gelpi, M. Povedano, G. Santpere, I. Ferrer, Gene Expression Profile in Frontal Cortex in Sporadic Frontotemporal Lobar Degeneration-TDP, J. Neuropathol. Exp. Neurol. 77, 608-627 (2018).

65. D. Martins-de-Souza, W. F. Gattaz, A. Schmitt, C. Rewerts, S. Marangoni, J. C. Novello, G. Maccarrone, C. W. Turck, E. Dias-Neto, Alterations in oligodendrocyte proteins, calcium homeostasis and new potential markers in schizophrenia anterior temporal lobe are revealed by shotgun proteome analysis, J Neural Transm (Vienna) 116, 275-289 (2009).

66. I. S. Piras, C. Bleul, I. Schrauwen, J. Talboom, L. Llaci, M. D. De Both, M. A. Naymik, G. Halliday, C. Bettencourt, J. L. Holton, G. E. Serrano, L. I. Sue, T. G. Beach, N. Stefanova, M. J. Huentelman, Transcriptional profiling of multiple system atrophy cerebellar tissue highlights differences between the parkinsonian and cerebellar sub-types of the disease, Acta Neuropathol Commun 8 (2020), doi:10.1186/s40478-020-00950-5. 
621 67. R. Tang, H. Liu, Identification of Temporal Characteristic Networks of Peripheral Blood 622 Changes in Alzheimer's Disease Based on Weighted Gene Co-expression Network Analysis, 623 Front Aging Neurosci 11, 83 (2019).

624 68. Z. Wang, X. Yan, C. Zhao, Dynamical differential networks and modules inferring disrupted 625 genes associated with the progression of Alzheimer's disease, Exp Ther Med 14, 2969-2975 626 (2017).

627 69. A. Lin, C. J. Giuliano, A. Palladino, K. M. John, C. Abramowicz, M. L. Yuan, E. L. Sausville, D. A. 628 Lukow, L. Liu, A. R. Chait, Z. C. Galluzzo, C. Tucker, J. M. Sheltzer, Off-target toxicity is a common 629 mechanism of action of cancer drugs undergoing clinical trials, Sci Trans/ Med 11 (2019), 630 doi:10.1126/scitranslmed.aaw8412.

631 70. S. Meier, M. Bell, D. N. Lyons, J. Rodriguez-Rivera, A. Ingram, S. N. Fontaine, E. Mechas, J. 632 Chen, B. Wolozin, H. LeVine, H. Zhu, J. F. Abisambra, Pathological Tau Promotes Neuronal 633 Damage by Impairing Ribosomal Function and Decreasing Protein Synthesis, J Neurosci 36, 634 1001-1007 (2016).

71. Y. Fujiki, A. L. Hubbard, S. Fowler, P. B. Lazarow, Isolation of intracellular membranes by means of sodium carbonate treatment: application to endoplasmic reticulum, J. Cell Biol. 93, 97-102 (1982).

72. M. M. Savitski, T. Mathieson, N. Zinn, G. Sweetman, C. Doce, I. Becher, F. Pachl, B. Kuster, M. Bantscheff, Measuring and managing ratio compression for accurate iTRAQ/TMT

Acknowledgements: We thank the UF Proteomics and Mass Spectrometry core for proteomic 643 analysis, the University of Kentucky Magnetic Resonance Imaging and Spectroscopy Center 644 (MRISC) for MRI analysis. We thank Dr. Peter Davies for his generous contribution of the 645 PHF1 antibody. We thank Dr. Fred Schmitt for insightful discussions and crucial intellectual 646 contributions to this study. We thank Dr. Shelby Meier for her contributions to the work prior to 647 this study and experimental support in this current project. Funding: This work was supported 648 by the Alzheimer's Association NIRG-14-322441, Department of Defense AZ140097, 649 NIH/NIMHD L32 MD009205-01, NIH 1R21NS093440, NIH/NINDS 1R01 NS091329-01. 650 Author Contributions: S.A.K., S.N.F., and J.F.A. designed the project. S.A.K., M.J.H., R.C., 
651 S.N.F., C.L., J.R.R., A.I., M.B., E.J.M., S.M.G.E., N.Z., N.S., D.T., D.P., M.V., T.F., and

652 A.M.S.H. performed experiments, acquired data, or assisted in analysis. S.A.K., M.J.H., and

653 J.F.A drafted the manuscript. J.K., J.M.A., and N.J.L. edited the manuscript. Competing

654 interests: GSK manufactured GSK2606414, which was used in this study. Moreover, this study

655 was funded in part by a contract from GSK. JMA and NL are employed by GSK. Data and

656 materials availability: Raw proteomic data will be accessible in PRIDE. 
Figures

Fig 1: Multi-target kinase inhibition with GSK2606414 rescues brain atrophy in rTg4510 tau transgenic mice.

661 A Timeline of tauopathy phenotype in transgenic ( $\mathrm{Tg}$ ) mice and depiction of drug delivery 662 between $4-5$ mo.

663 B-E Representative T2-weighted MR images of non-transgenic (NTg) and Tg mice treated with 664 vehicle (Veh) or GSK2606414 (414).

665 F Total brain volume quantification $(\mathrm{NTg}+414, \mathrm{n}=2$; others $\mathrm{n}=3)$. Two-way ANOVA with 666 Tukey post hoc test. Data are expressed as the mean \pm SEM, **p $<0.01$.

Fig 2: GSK2606414 treatment rescues brain dysfunction in tau transgenic mice.

A-D Representative DR1-rendered MEMRI images. The insets in panels A-D show half of the

671 E-F Calculated DR1 values in the dentate gyrus (DG, E) and Cannu ammonis 1 (CA1, F). Two-

672 way ANOVA with Tukey post hoc test. Data are expressed as the mean $\pm \mathrm{SEM}, * p<0.05, *^{*} p<$

$6730.01, * * * p<0.001,(\mathrm{n}=4-5)$.

674 G Results of open field behavioral task indicating percent of time spent in the periphery, normalized to the first five minutes $(\mathrm{n}=6-10)$. Two-way ANOVA with Tukey post hoc test.

676 Data are expressed as the mean relative to NTg + Veh \pm SEM, $* p<0.05$.

677 H Y-maze behavioral task results for number of novel arm entries ( $\mathrm{n}=5-7)$. Two-way ANOVA 678 with Tukey post hoc test. Data expressed as the mean \pm SEM, *p $<0.05$, \& denotes $\mathrm{p}=0.1095$.

Fig 3. GSK2606414-mediated cognitive rescue in early-stage tau transgenic mice is independent of tau hyper-phosphorylation and GSK3 $\beta$ activity

682 A Immunoblots of PHF1 (S396/S414) and total tau in 5mo NTg and Tg animals treated with 683 vehicle or GSK2606414 (414).

684 B-D Results of PHF1 (B), total tau (C), and relative phosphorylation (D) is normalized to 685 GAPDH $(\mathrm{n}=3)$. Two-way ANOVA with Tukey post hoc test. Data are expressed as the mean 686 relative to $\mathrm{Tg}+\mathrm{Veh} \pm \mathrm{SEM}$.

687 G-J Representative images of active, pT980 PERK (pPERK) staining. All images were post688 processed equally and highlights lack of positive staining in $5 \mathrm{mo} \mathrm{Tg}$ mice. Scale bar $=25$ 689 microns.

690 K Quantification of pPERK staining reveals that 414 treatment has no detectable change in 5 mo 691 Tg mice $(n=5-7)$, but strongly inhibits PERK activity in the CA3 and superior medial cortex 
692 (Ctx) in 8mo Tg mice (hatched bars, $\mathrm{n}=3$ ). Two-way ANOVA with Sidak post hoc test. Data are expressed as the mean relative to NTg + Veh \pm SEM, $* * * * p<0.0001$.

L Relative hippocampal gene expression of direct mediators of the PERK-UPR pathway in 5 mo NTg and Tg mice treated for 30d with Veh or 414 normalized to GAPDH and $18 \mathrm{~S}(\mathrm{n}=4-6)$. Two-way ANOVA with Tukey post hoc test. Data are expressed as the mean relative to NTg + Veh \pm SEM, $* \mathrm{p}<0.05, * * \mathrm{p}<0.01$.

M-N Representative images (M) and quantification (N) of overlap between NRF2 (red) and nuclear staining (DAPI, blue) in the CA3 of 5mo NTg and Tg mice treated with either Veh or $414(\mathrm{n}=3)$. Unpaired Student's t-test. Error bars denote SEM. Scale bar $=25$ microns.

Fig 4. Hippocampal proteome signatures of early stage tauopathy model mice.

A Schematic of TMT isobaric tag proteomic pipeline. Mice hippocampal sections were extracted and lysed. Individual isobaric tags were used to chemically label proteins from each individual sample and combined for LC/MS-MS detection. Batch normalization was completed using a shared batch correction standard, resulting in $\mathrm{n}=4$ per group. All samples were from female mice.

B Heatmap of proteomics results showing 337 significantly altered proteins across all comparisons (FDR adjusted, $\mathrm{p}<0.05$ ).

710 C Summation of the number of significantly altered proteins across each test.

711 D Representative protein for each test of significant differential abundance.

712 E Protein abundance of human MAPT P301L transgene.

713 F Abundance of each human MAPT P301L phospho-peptide detected by phospho-proteomics.

714 G-H Network clustering of Reactome pathways found up-regulated (G) or down-regulated (H) due to tau transgenic genotype relative to NTg mice (Tg effect). Node size proportionally represents the protein count in that pathway, whereas node color represents the statistical significance gradient (FDR-adjusted $\mathrm{p}$ value) from $\mathrm{p}=0.10$ (brown) approaching $\mathrm{p}=0$ (white).

Fig 5. Multi-target kinase inhibition rescues pathways related to cognitive and neuronal Dashed connections indicate manually adjusted nodes.

722 A-B Network clustering of Reactome pathways found up-regulated (F) or down-regulated (G) in $723 \mathrm{Tg}$ mice and significantly rescued toward NTg + Veh levels in $\mathrm{Tg}+414$ mice. Node size proportionally represents the protein count in that pathway, whereas node color represents the statistical significance gradient (FDR-adjusted $p$ value) from $p=0.10$ (brown) approaching $p=0$ (white). Dashed connections indicate manually adjusted nodes. absolute integrated density \pm SEM, $* p<0.05$. Scale bar $=25$ microns. 
E-F Immunoblots (e) of total tyrosine nitration and total lane quantified results $(\mathbf{F})$ relative to NTg + Veh and normalized to actin $(n=3-4)$. Two-way ANOVA with Tukey post hoc test. Data are expressed as the mean relative to $\mathrm{Tg}+\mathrm{Veh} \pm \mathrm{SEM}$, ** $\mathrm{p}<0.01$.

Fig 6: Proteomic signatures of rTg4510 transgenic mice match human tauopathy and reveal novel candidates for therapeutic intervention.

A-B Significantly altered proteins by Tg effect were compared to human homolog abundances found in two proteomics datasets of human neurodegenerative diseases (A, (37); B, (39)). Proteins annotated in the "Rescued" column (yellow) were significantly rescued by 414 in $\mathrm{Tg}$ animals compared to NTg controls. Human homolog protein abundances in each disease group were compared to non-demented controls. Statistically altered human proteins were annotated by disease group in different colors (Asym AD, pink; late AD, purple; late PD, green). Proteins that were significantly altered in the same direction (increased or decreased) between species and also represented proteins rescued by 414 in Tg mice were annotated in the "Consistent" column (black). Candidate proteins passing all criteria for inclusion were labeled with their corresponding human gene symbols. Sample abundances were averaged per group and each corresponding sample number was appended to the bottom of each column.

748 C Venn diagram comparing candidate proteins identified in late AD across both datasets.

\section{Supplementary Materials}

750 Supplementary Materials and Methods

751 Fig. S1. Total duration spent in novel arm is partially rescued in tau transgenic mice treated with 752 GSK2606414.

Fig. S2. Non-PERK UPR proteins do not have altered transcript levels in 5 mo tau transgenic mice.

760 Table S2. Significantly enriched reactome pathways identified by proteomics.

761 Table S3. Human to mouse proteomic comparisons.

762 Table S4. Patient demographics of human proteomic samples.

763 References $(71,72)$ 
A

Age

(months) 0

1

2

3

GSK2606414

Progressive tau pathology

Cognitive impairment

Significant brain atrophy
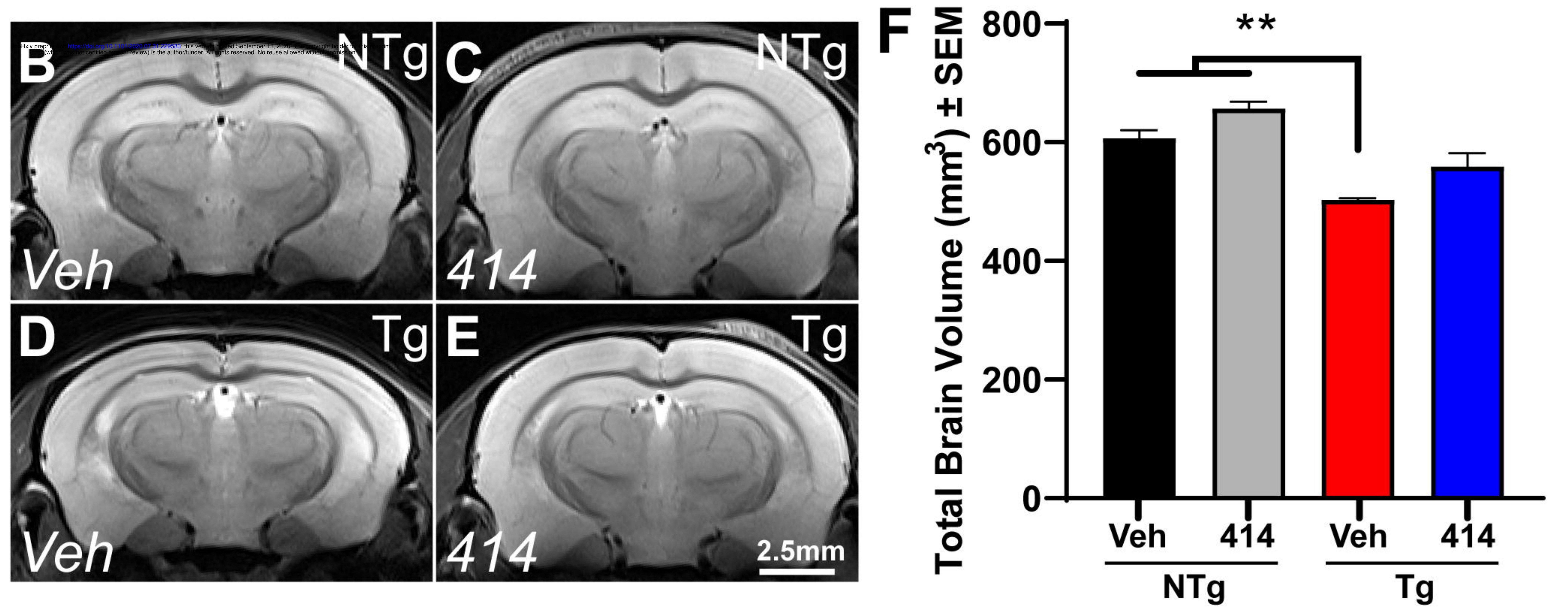


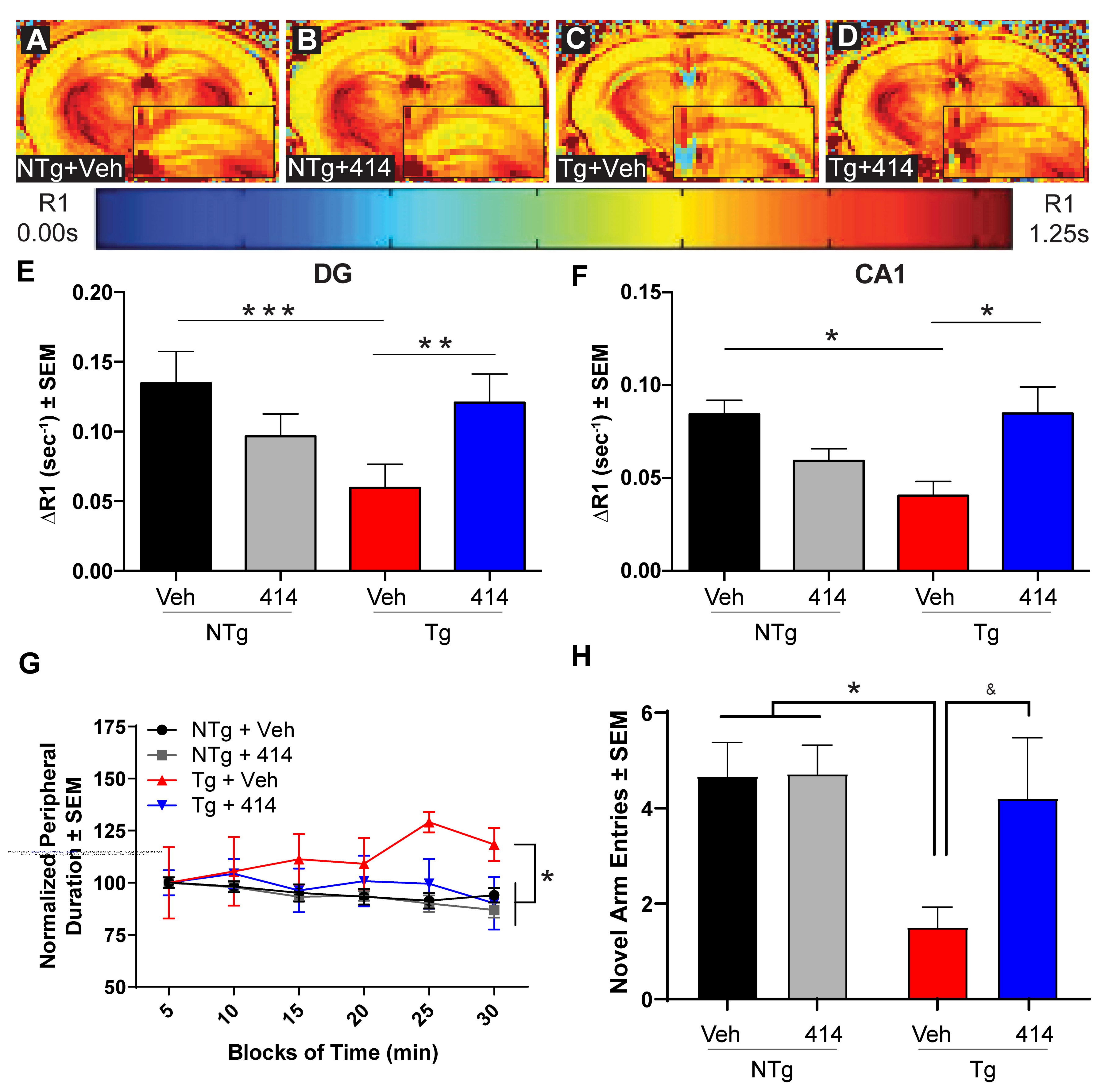


A Hippocampal protein extraction

TMT labelling

$\mathrm{NTg}+\mathrm{Veh}$
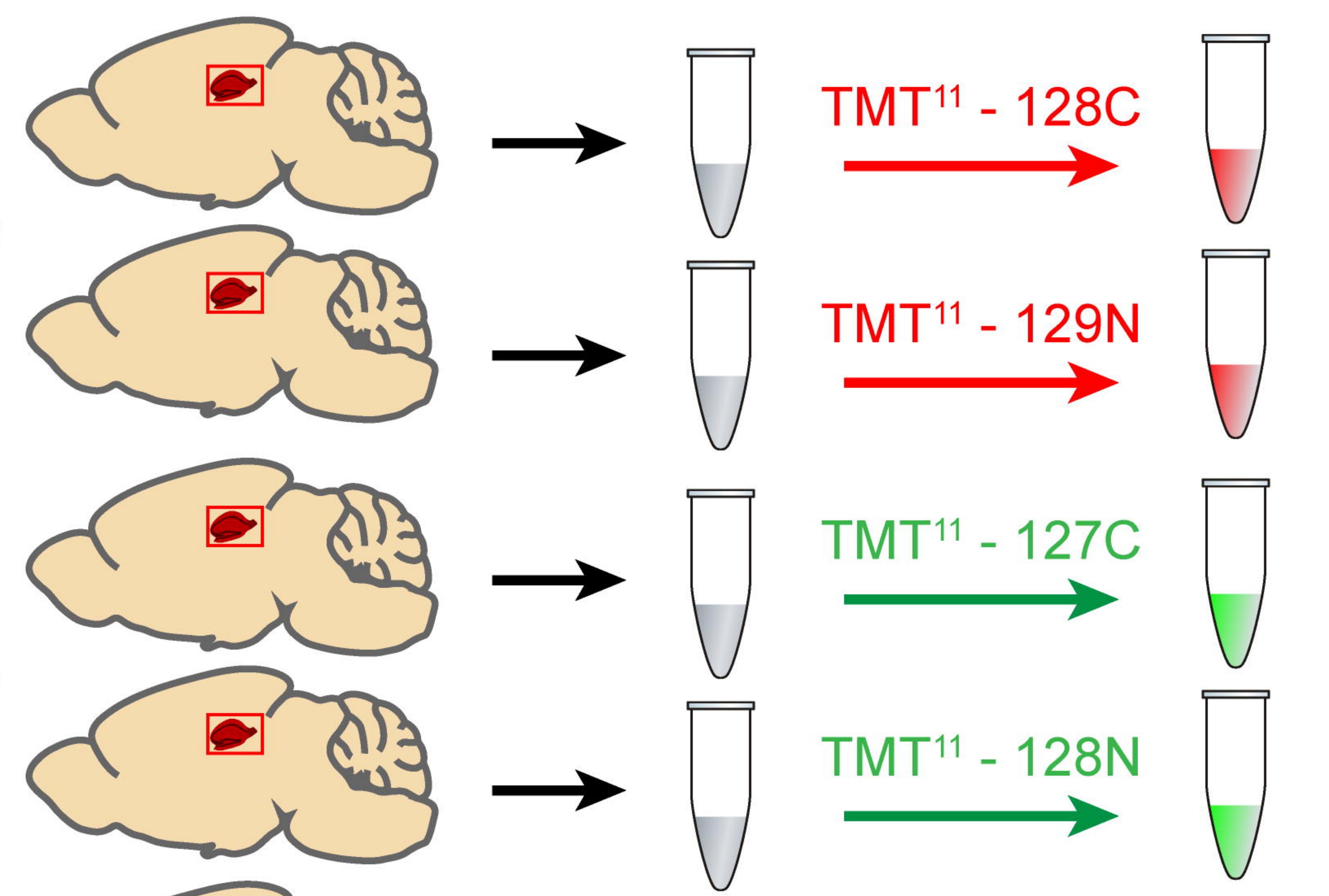

$\mathrm{Tg}+\mathrm{Veh}$
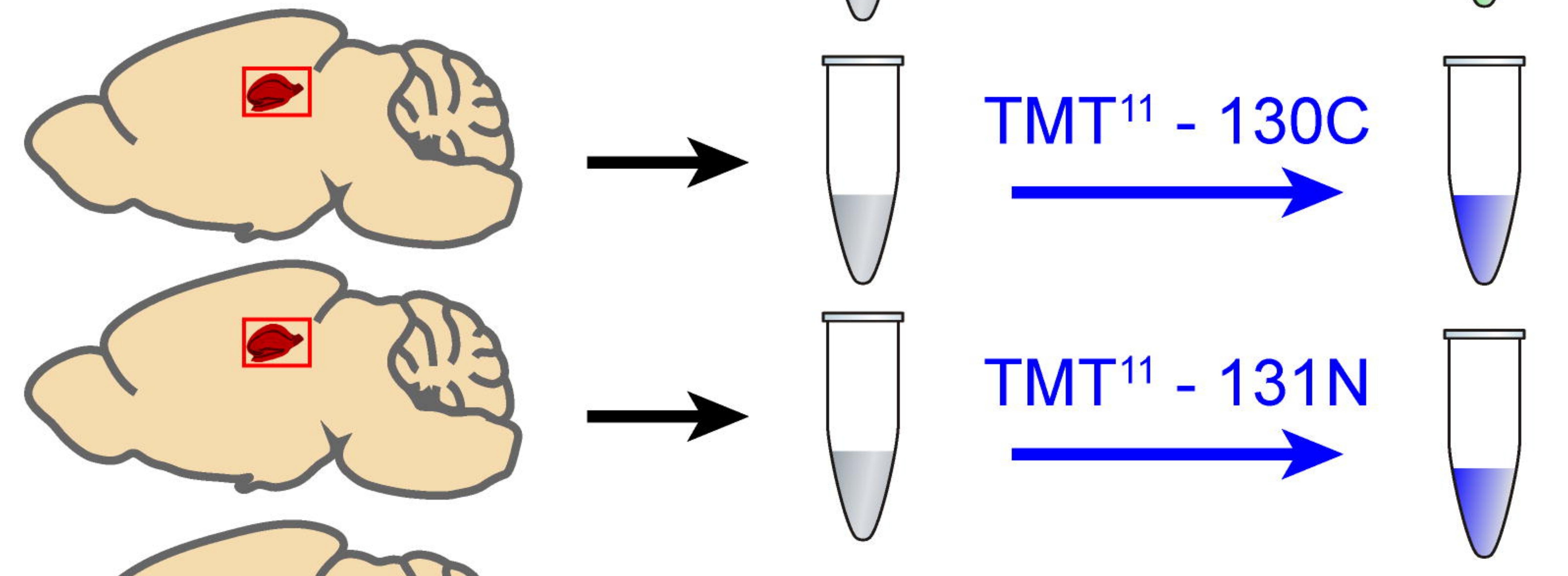

$\operatorname{Tg}+414$
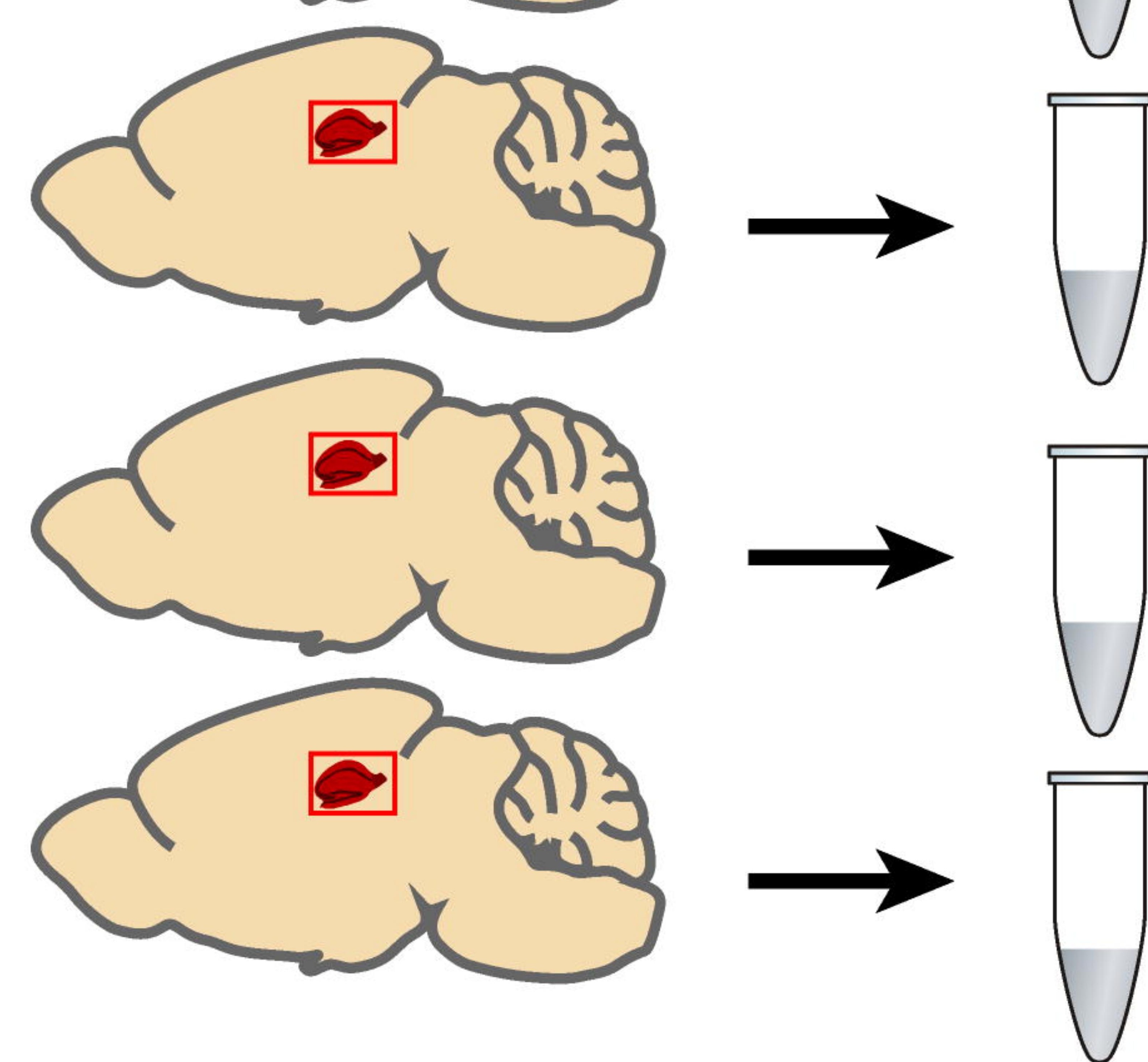

Batch Correction Standard

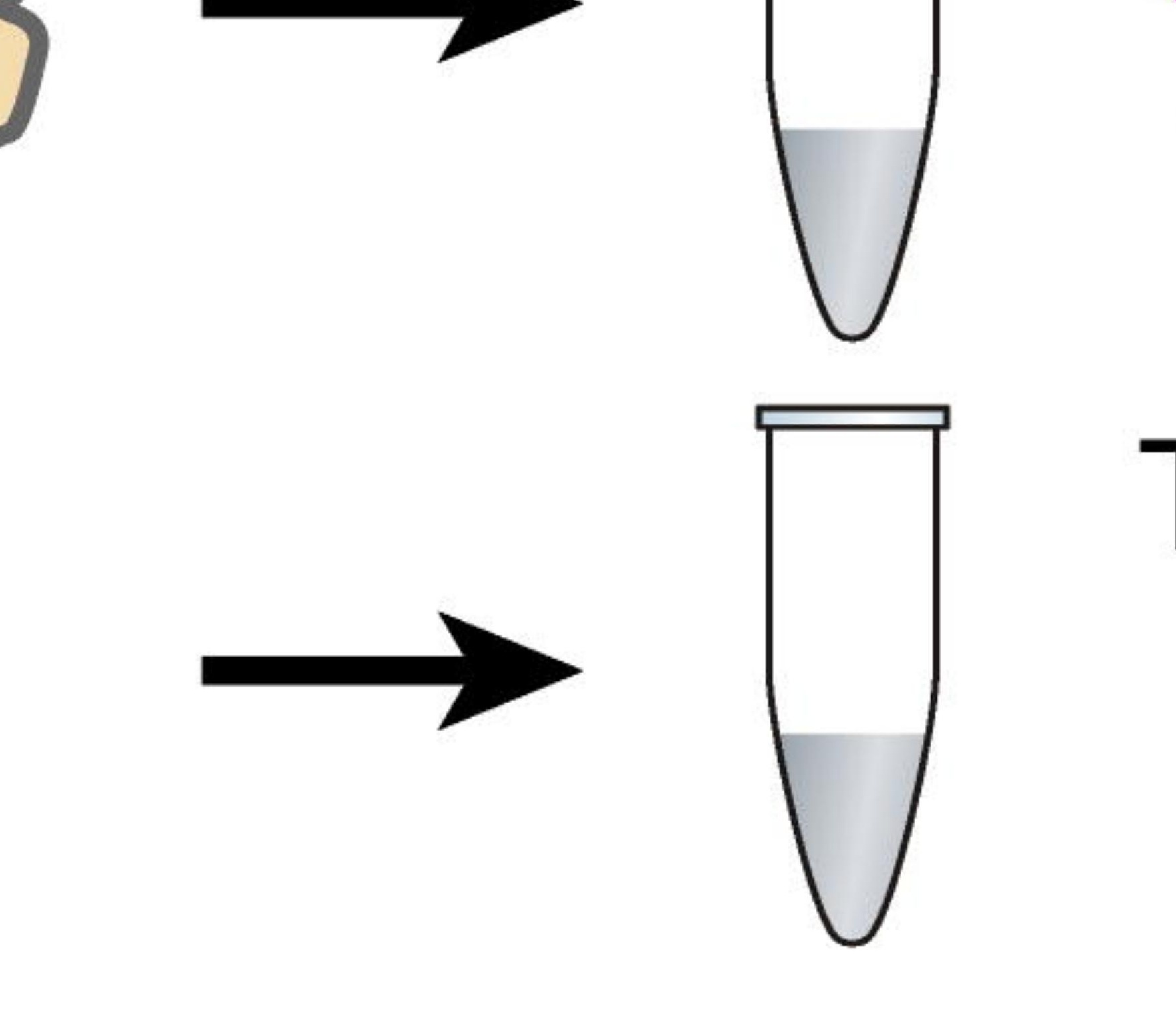

$\stackrel{\mathrm{TMT}^{11}-131 \mathrm{C}}{\longrightarrow}$

G

\section{Down-regulated in $\mathrm{Tg}$}

Synaptic Activity and LTP

mTOR Pathway

Activity
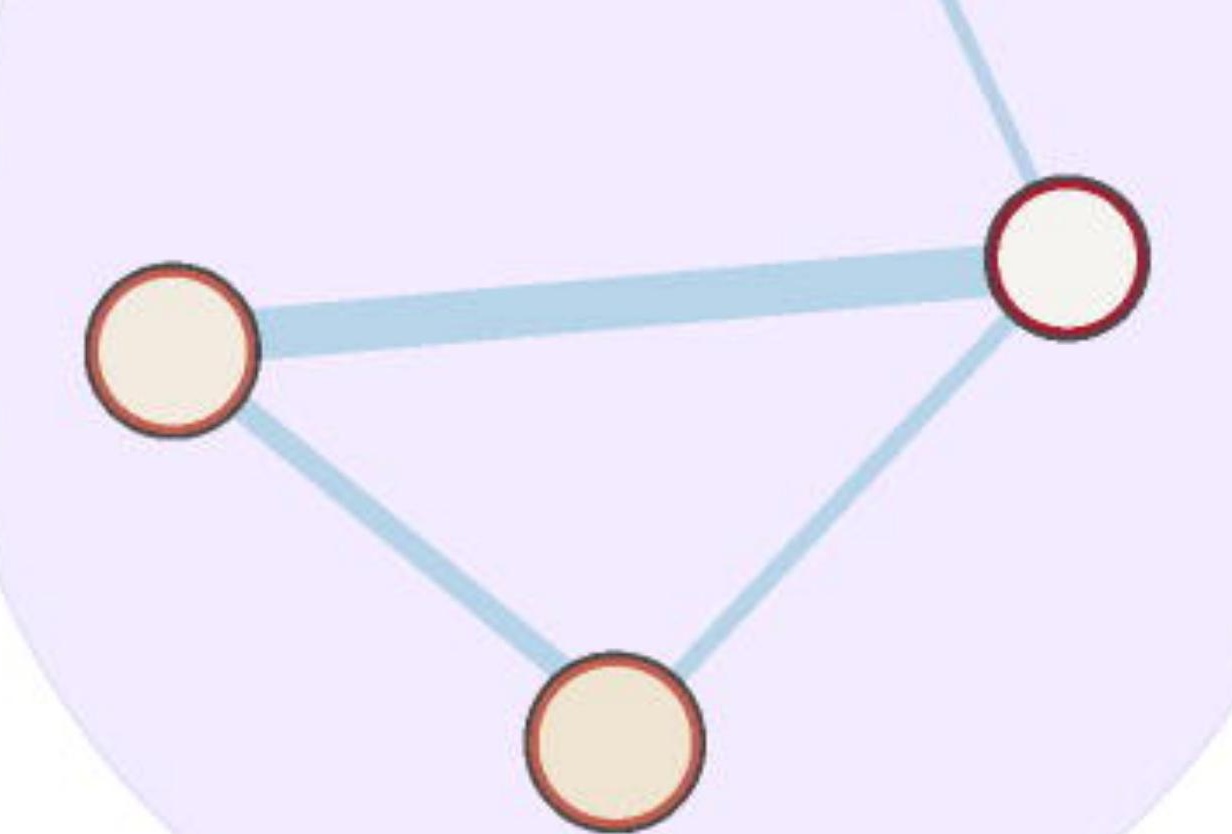

esicle-mediated Transport and Endocytosis

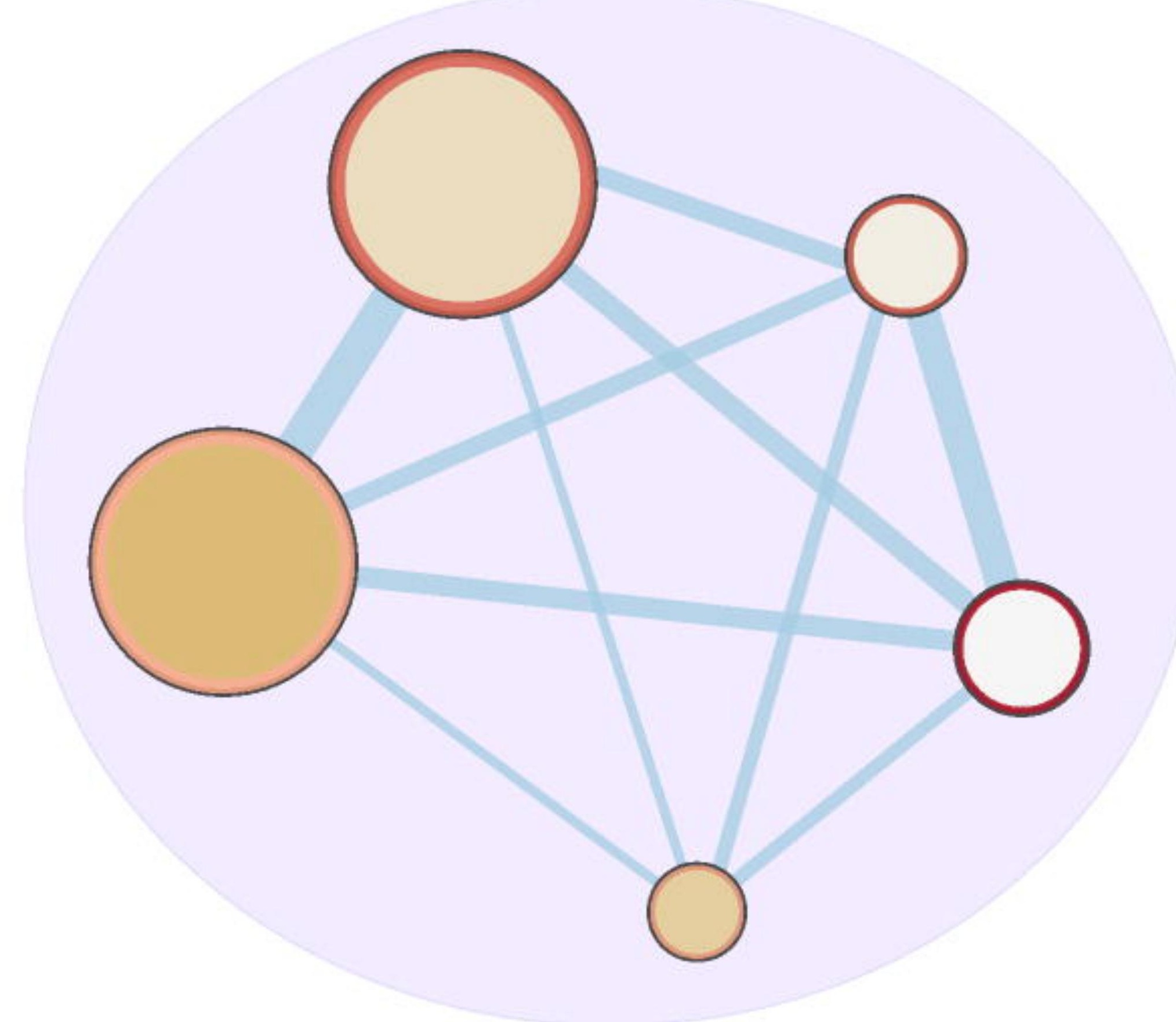

RNA Metabolism

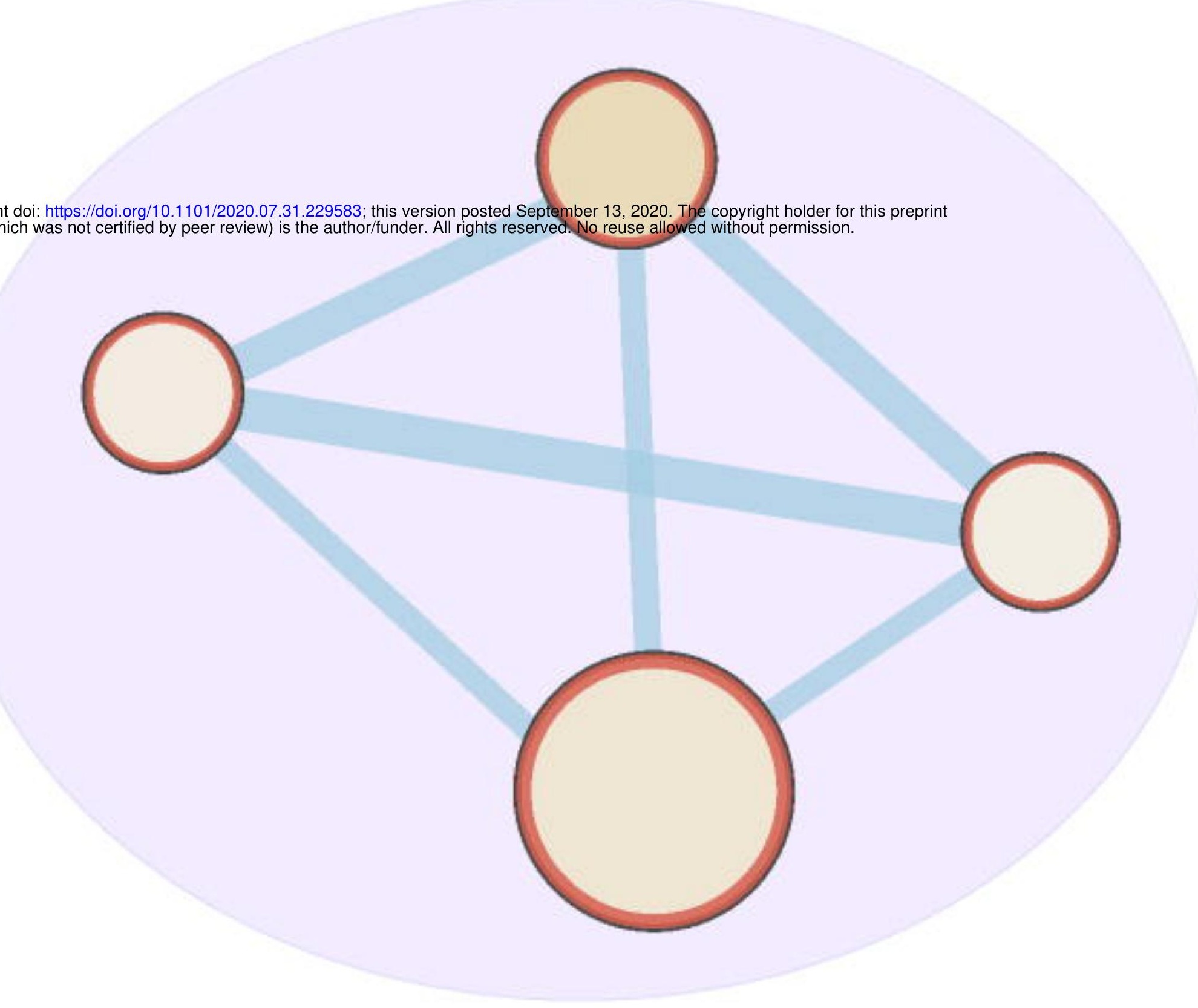

$\mathrm{NTg}+414$

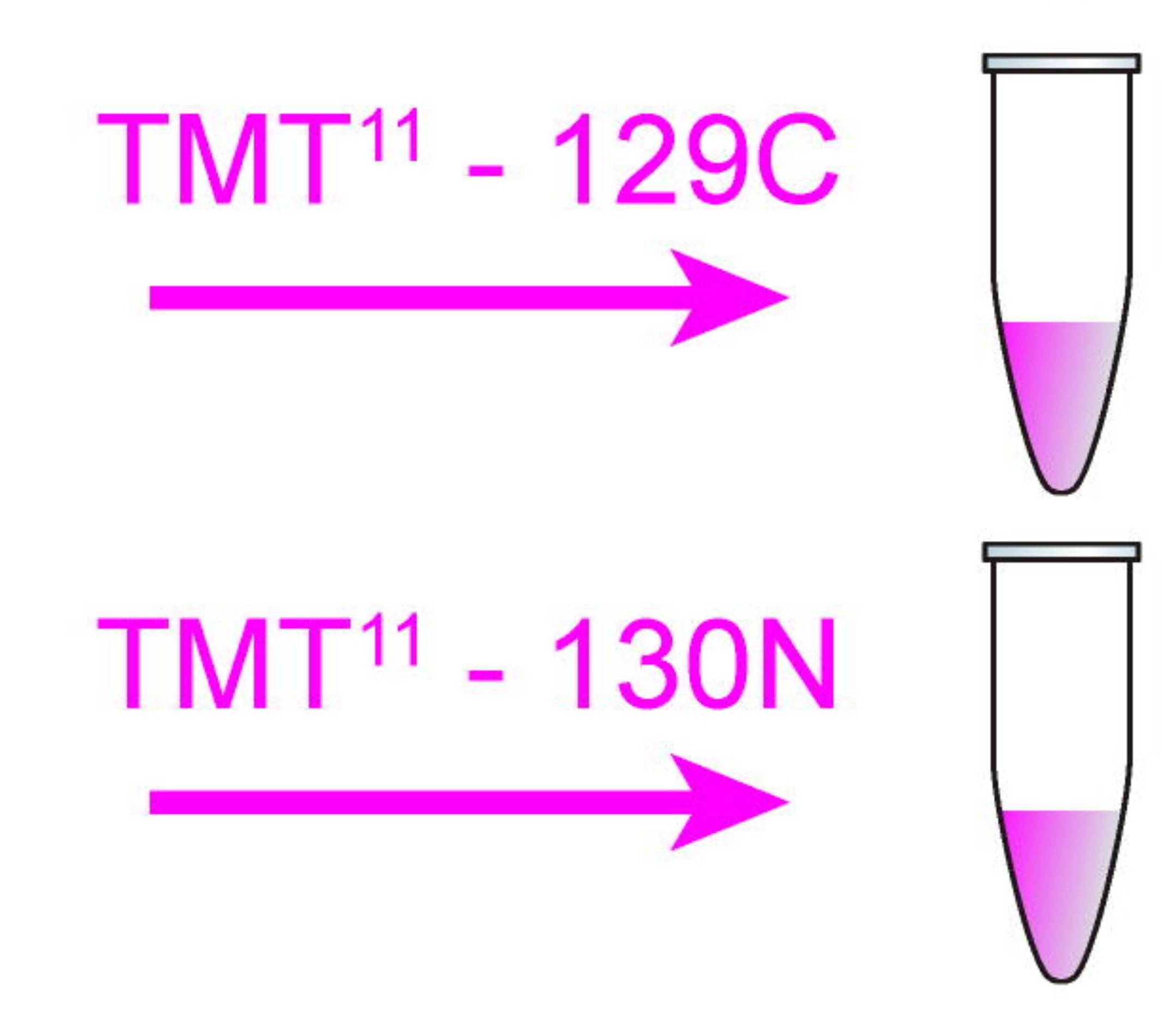
and Splicing
ROS Cofactor Defense
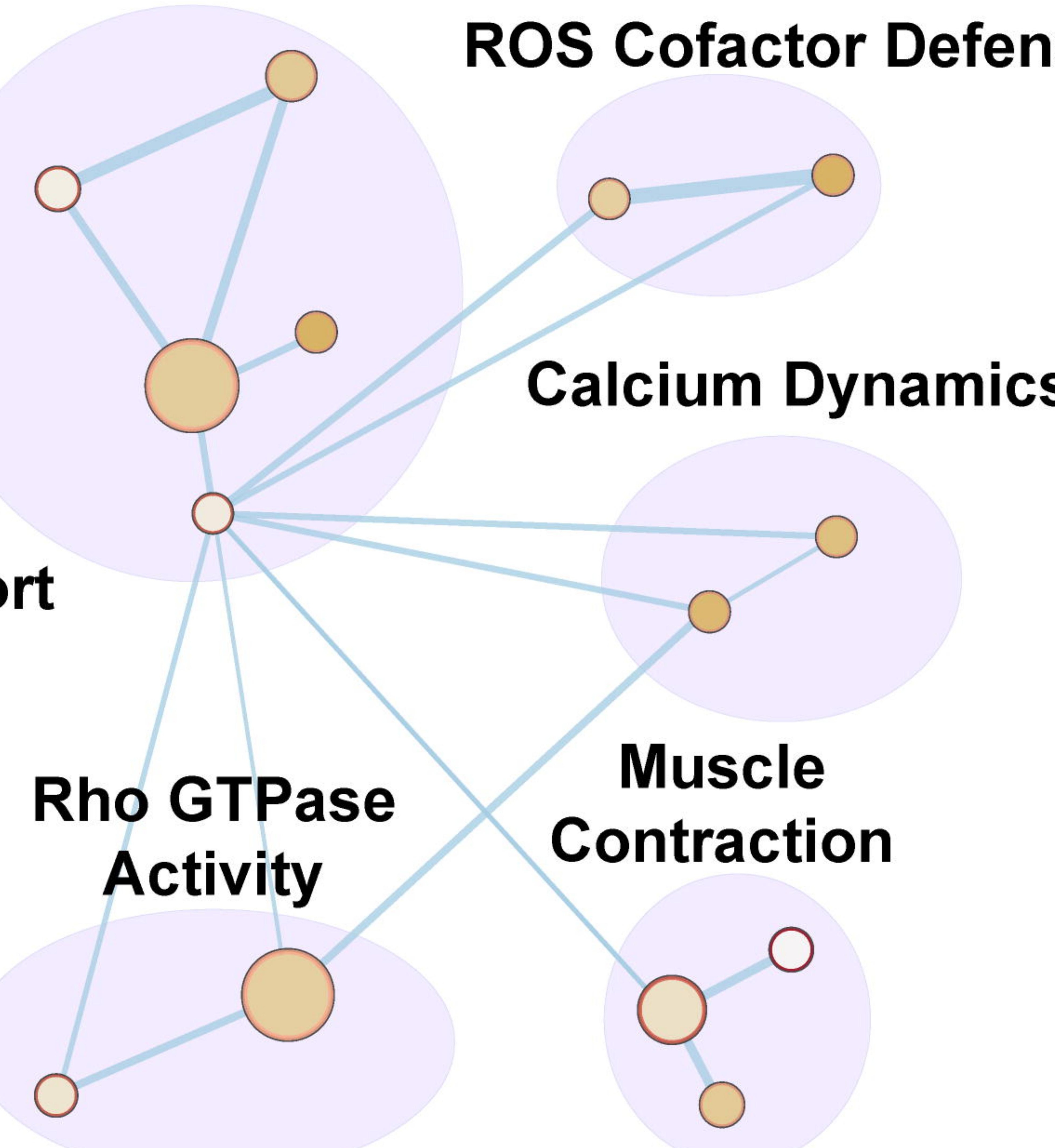

Aerobic Respiration and Mitochondrial Function

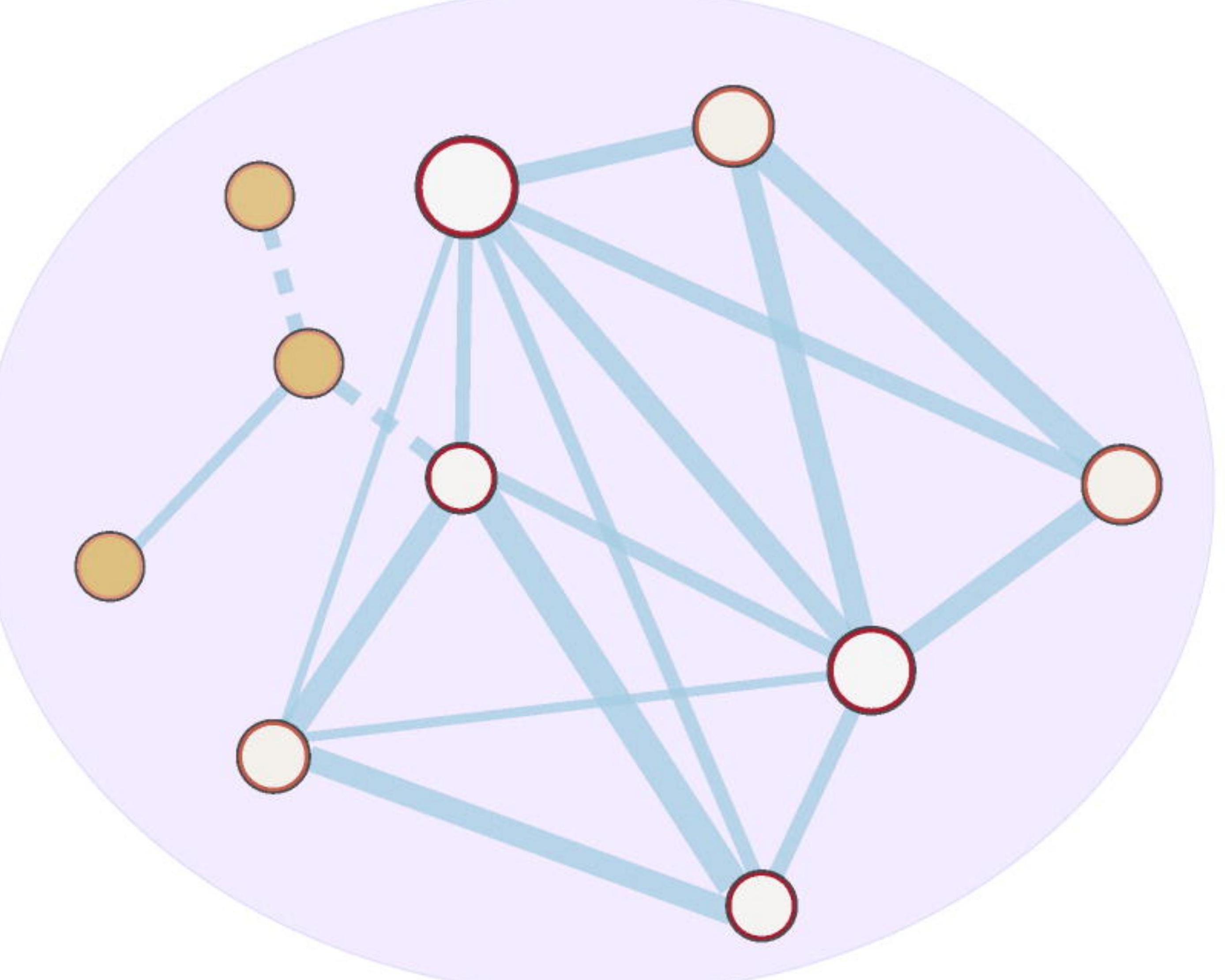

Pool peptides,

LC-MS/MS,

mouse Uniprot search

$2 x$ batches $(n=4)$,

batch normalization,

statistical analysis
B $\quad \mathrm{NTg} N T g \quad \mathrm{Tg} \quad \mathrm{Tg}$

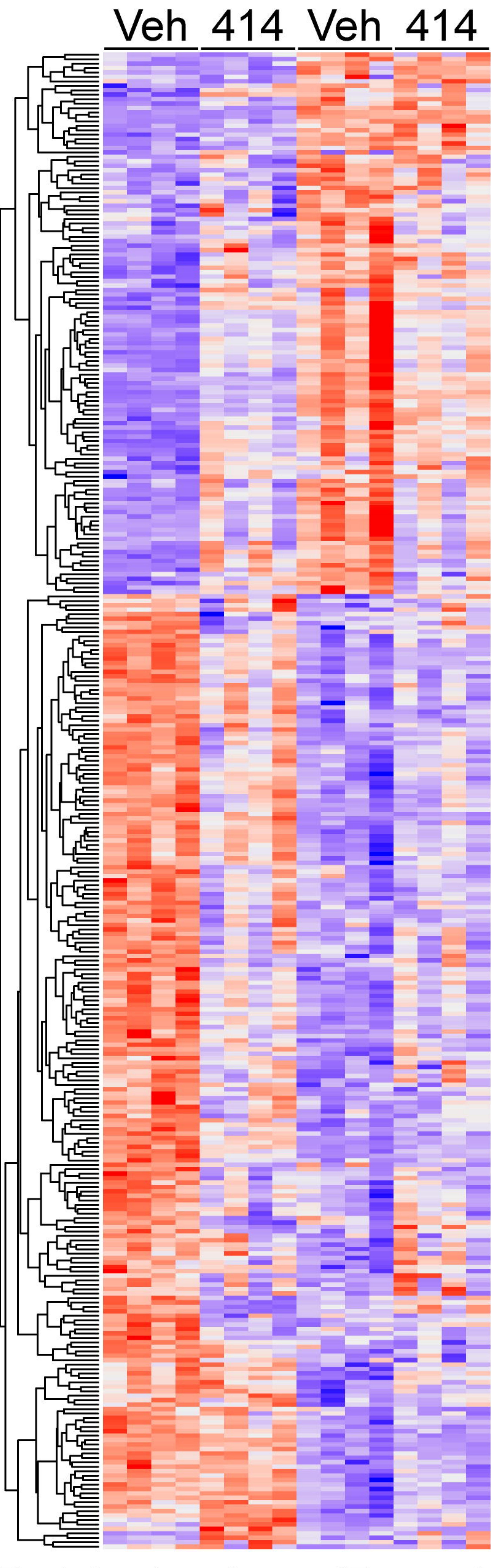

Protein abundance (Z-score)

$\begin{array}{rrrrr}-2 & -1 & 0 & 1 & 2\end{array}$
C

\begin{tabular}{|r|r|r|r|}
\hline \multirow{2}{*}{} & \multicolumn{3}{|c|}{$\begin{array}{c}\text { \# Proteins Significant } \\
\text { (FDR adj. p val < 0.05) }\end{array}$} \\
\cline { 2 - 4 } & \multicolumn{1}{|c|}{ Up } & \multicolumn{1}{c|}{ Down } & \multicolumn{1}{c|}{ Total } \\
\hline Drug Effect & 21 & 14 & 35 \\
\hline Tg Effect & 92 & 184 & 276 \\
\hline Drug Rescued & 66 & 120 & 186 \\
\hline
\end{tabular}

D Selected Representative Proteins

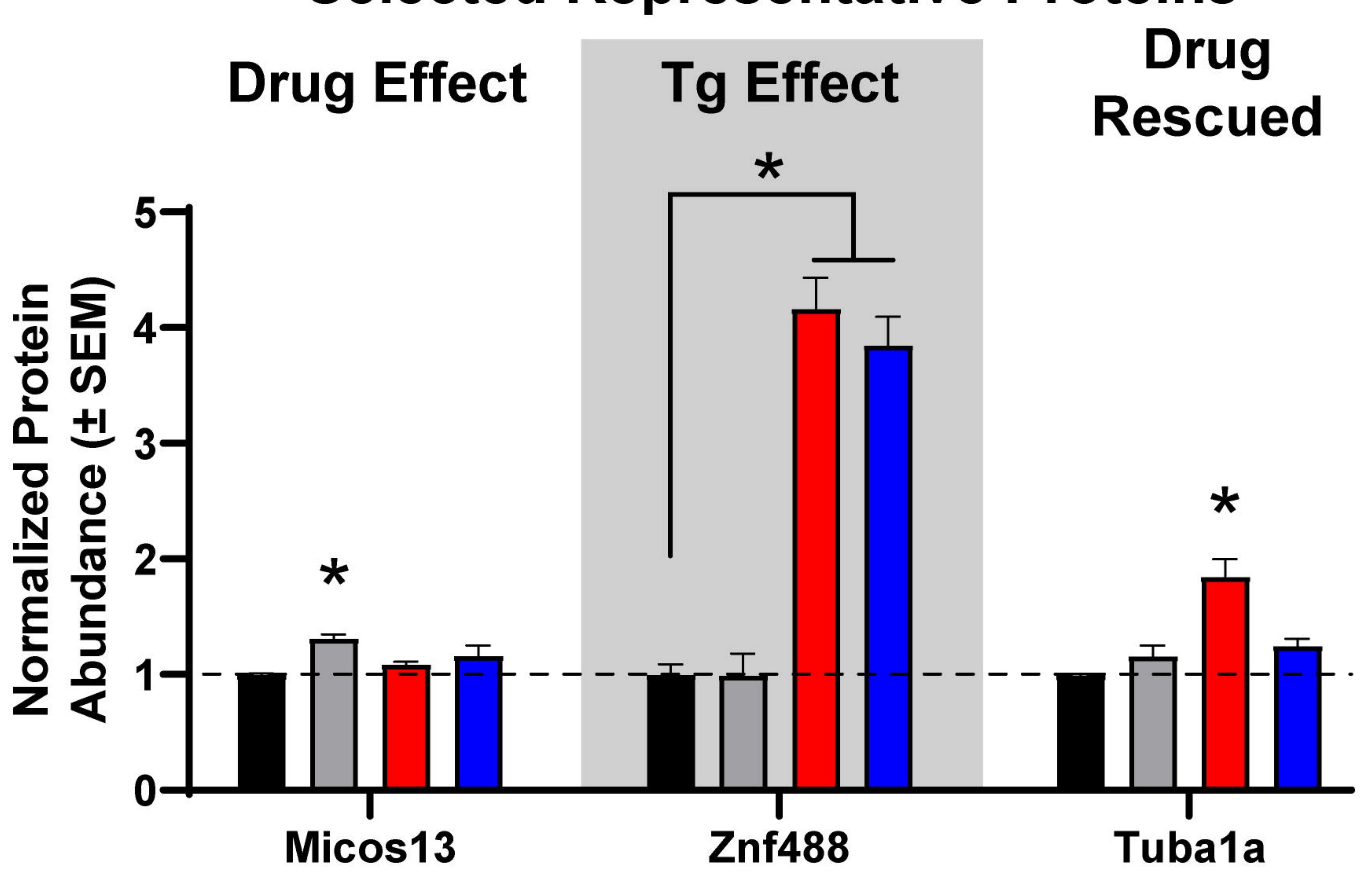

E

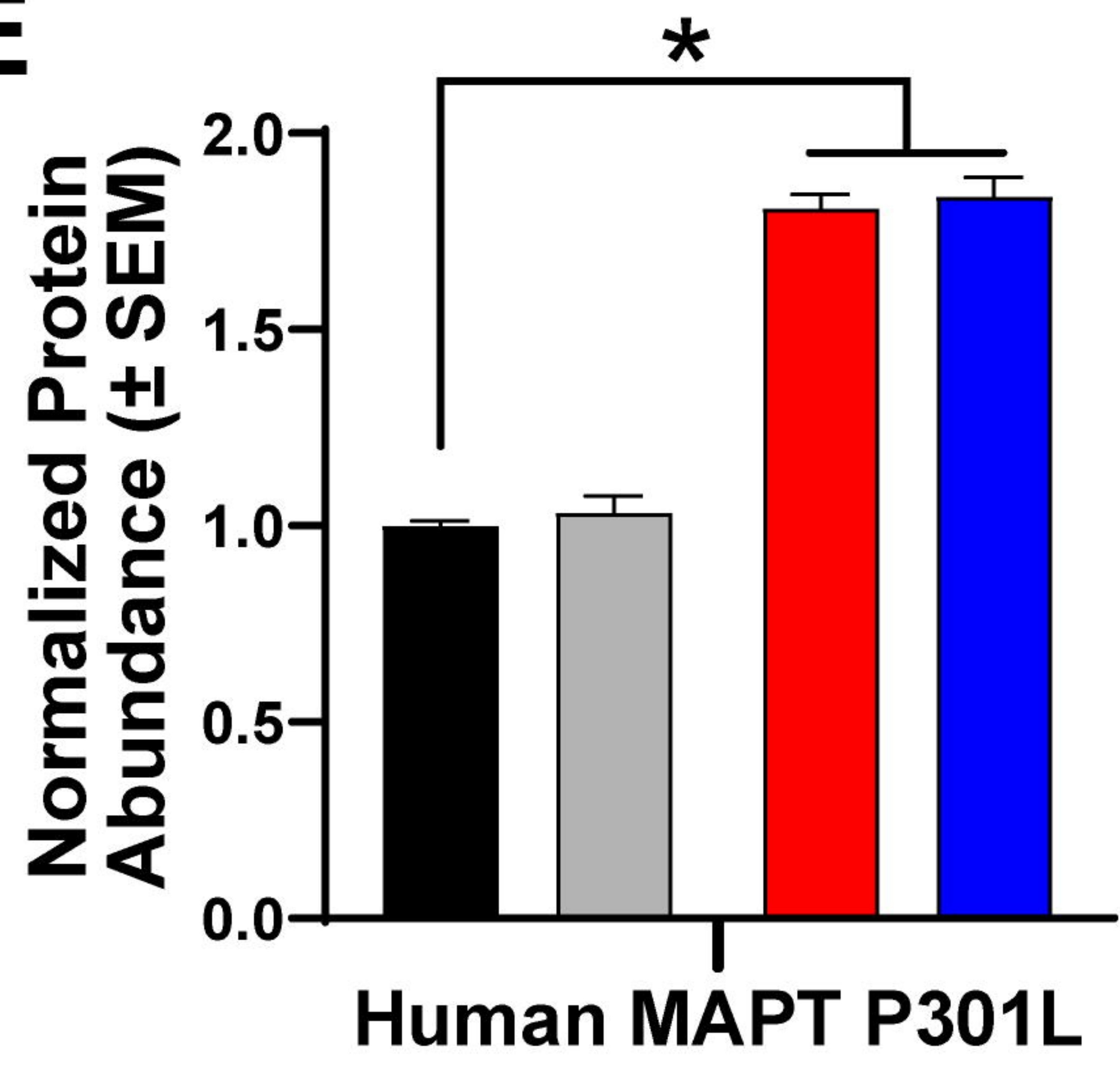

$\mathbf{F}$

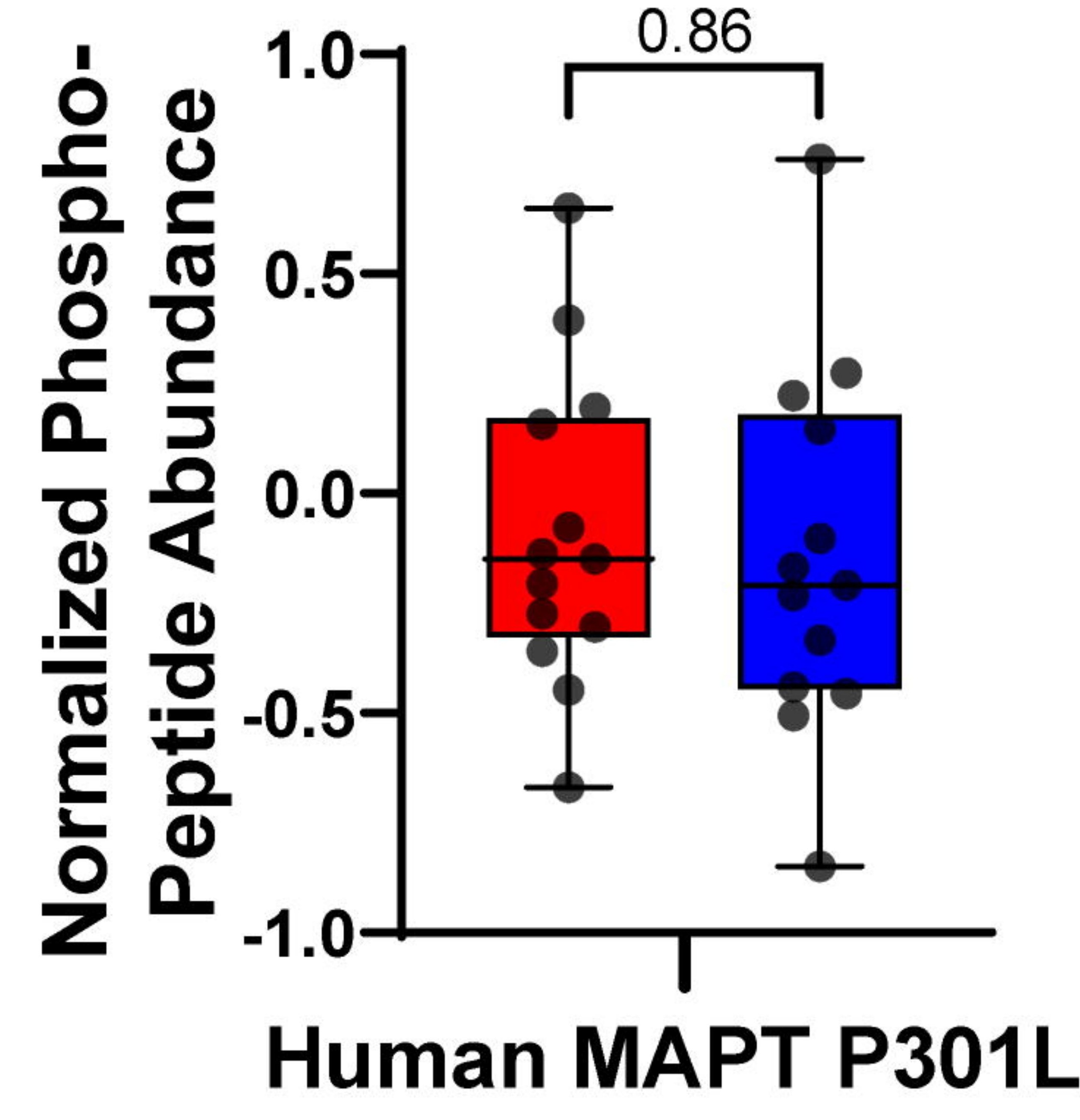

H

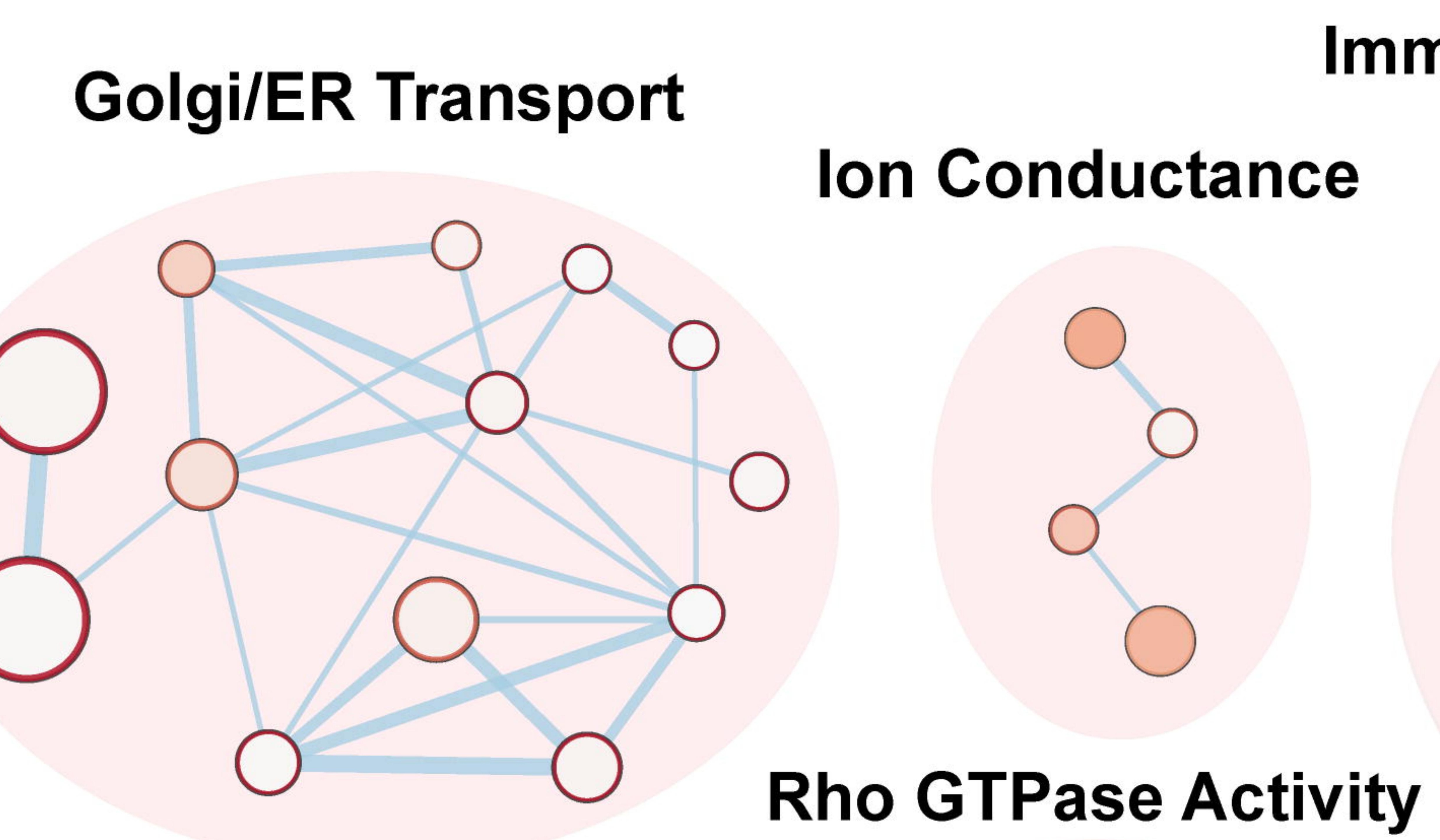

Gap junctions and Plasma Membrane

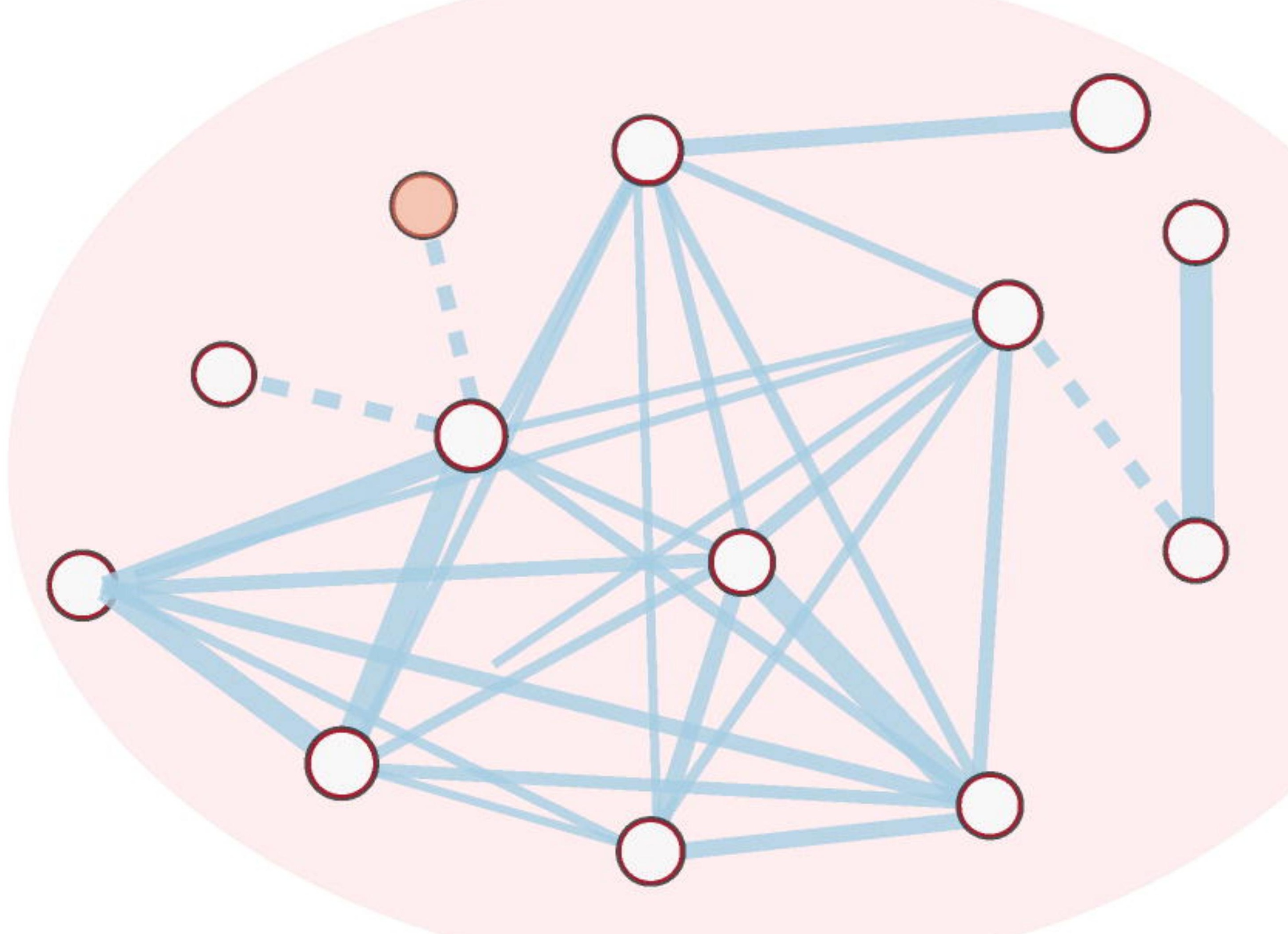

Wnt and Hedgehog

\section{Up-regulated in Tg}

Immune Response Kinase Activity

$\begin{array}{ll}\mathrm{NTg}^{+} \text {Veh } & \square \mathrm{Tg}+\text { Veh } \\ \square \mathrm{NTg}+414 \quad \square \mathrm{Tg}+414\end{array}$

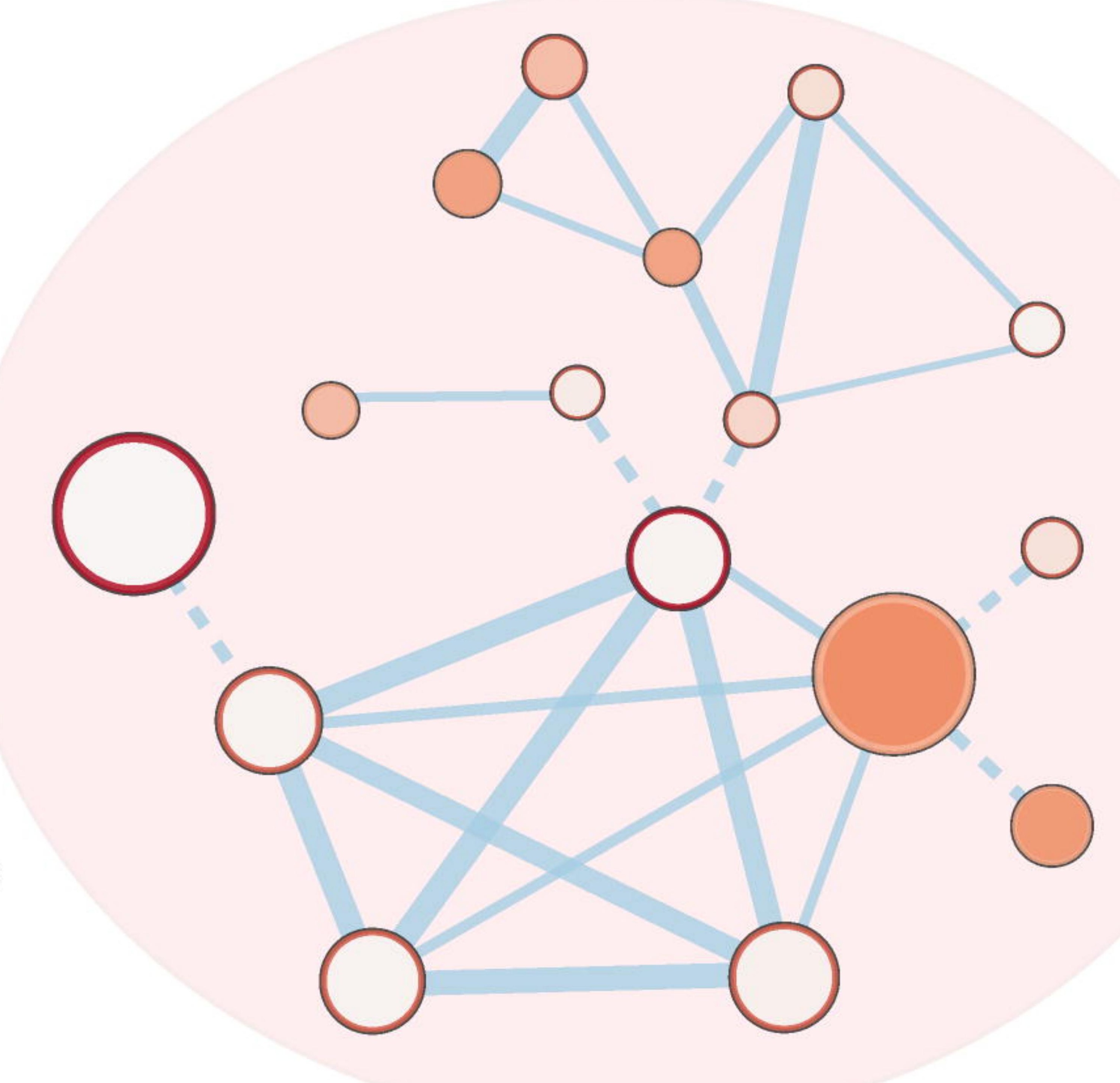

Aerobic Respiration and Mitochondrial Function

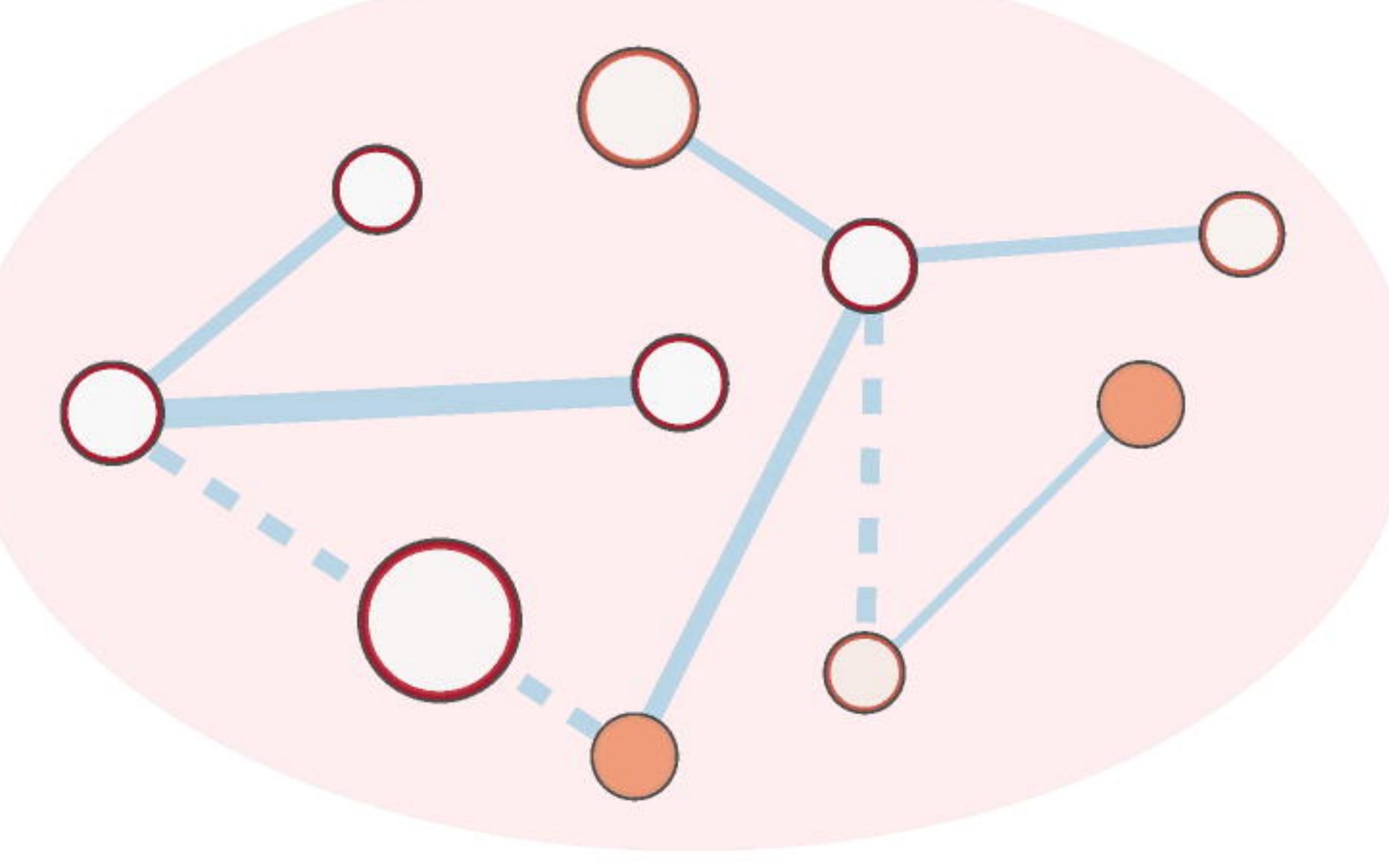

Rho GTPase Activity

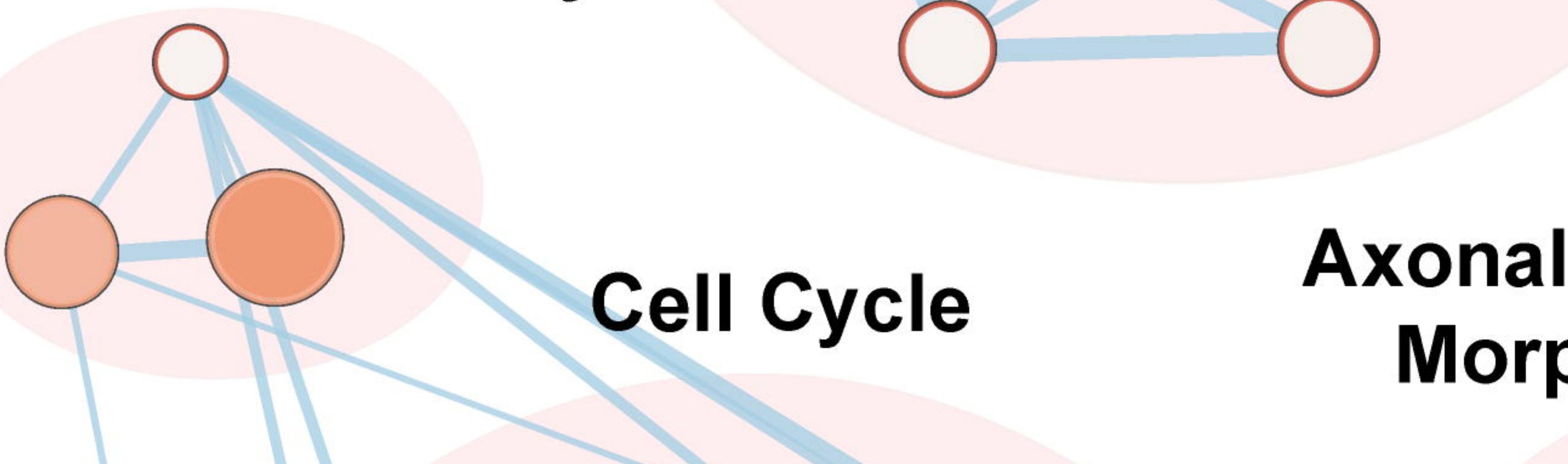

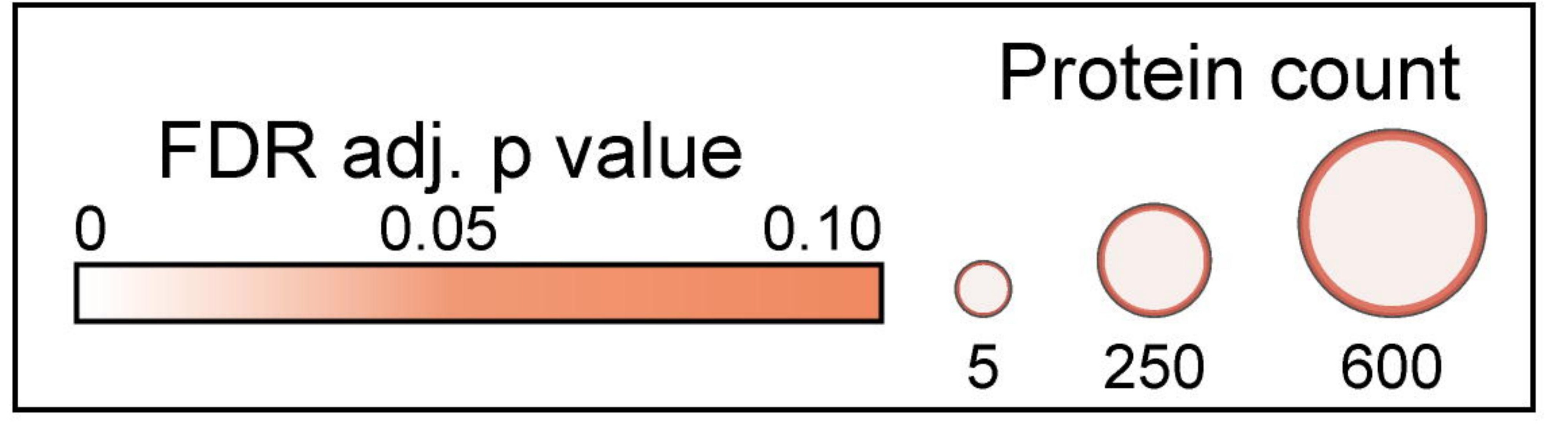
Signaling

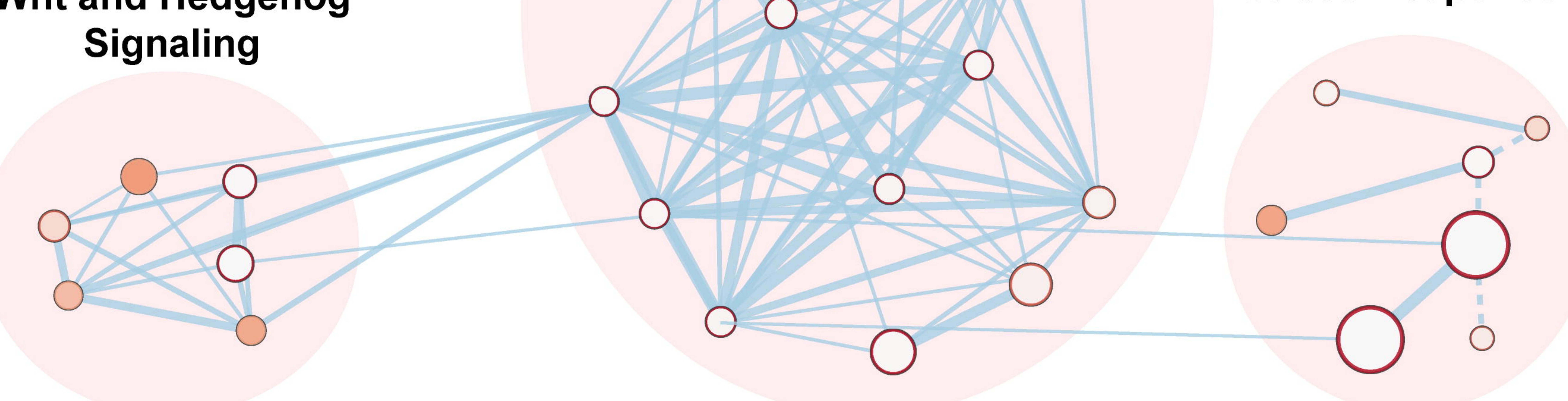

rotransmitter Release

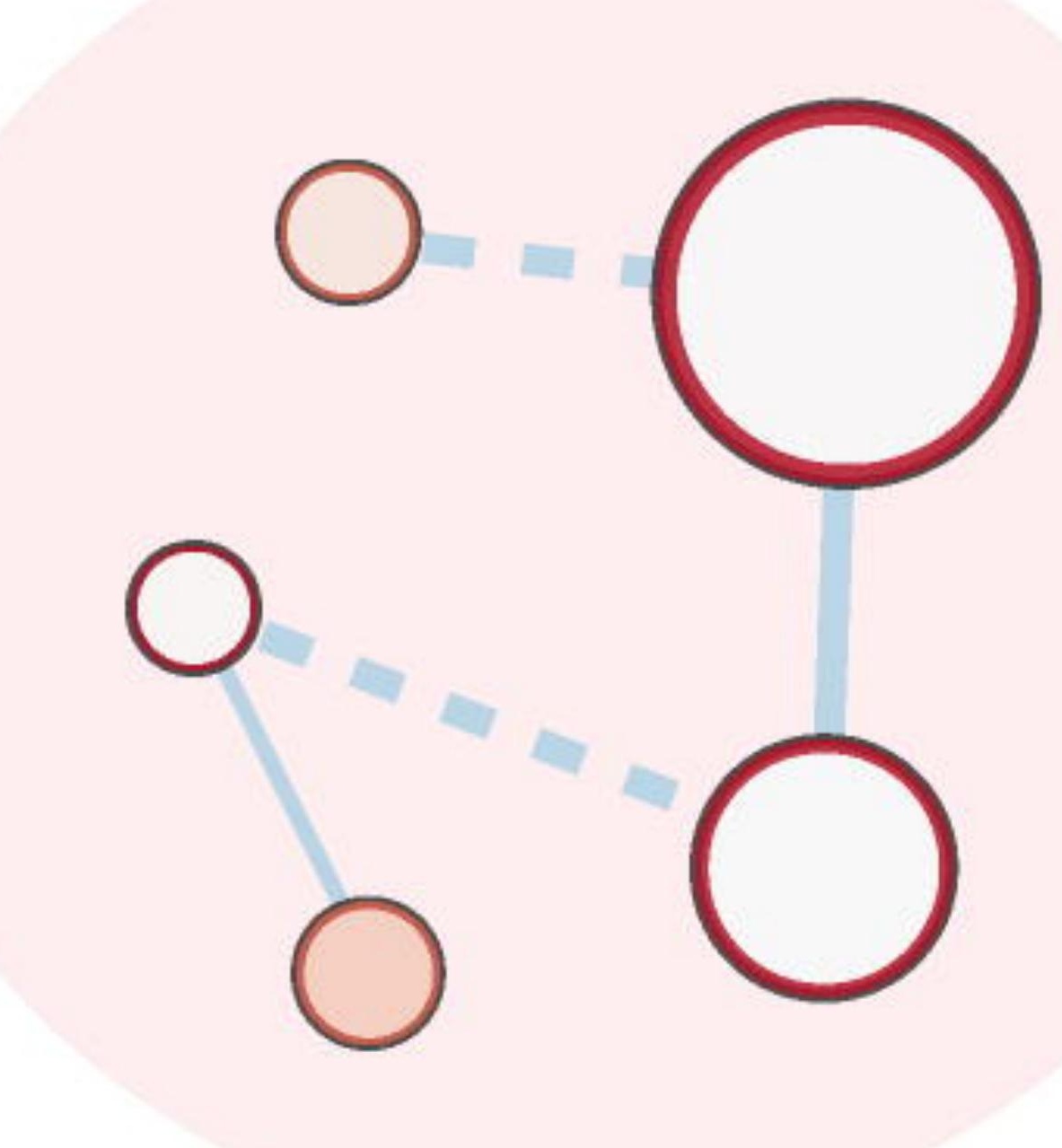

Heat Shock Stress Response o

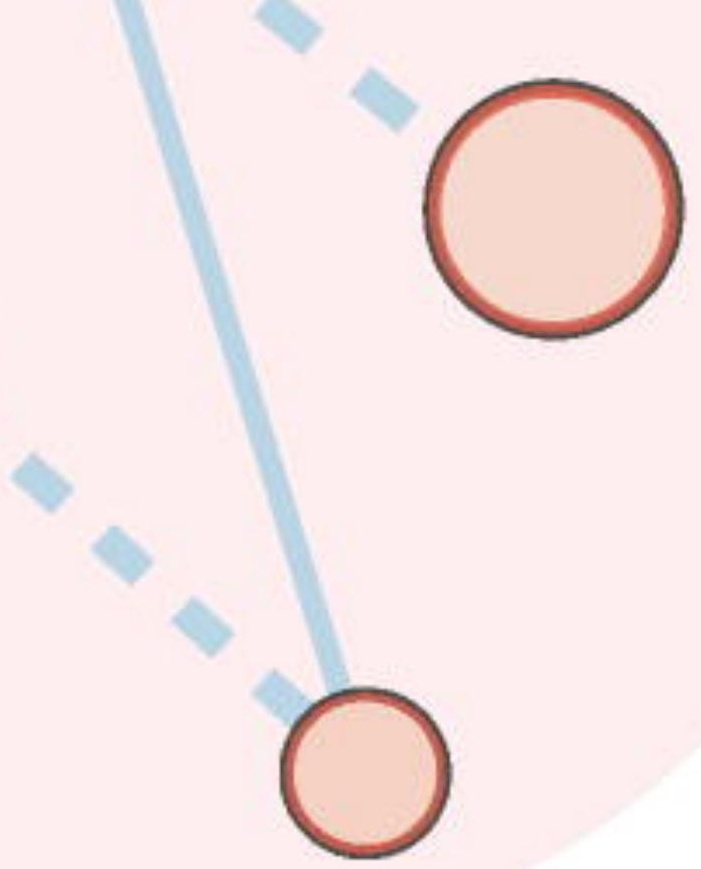

Amino Acid Metabolism

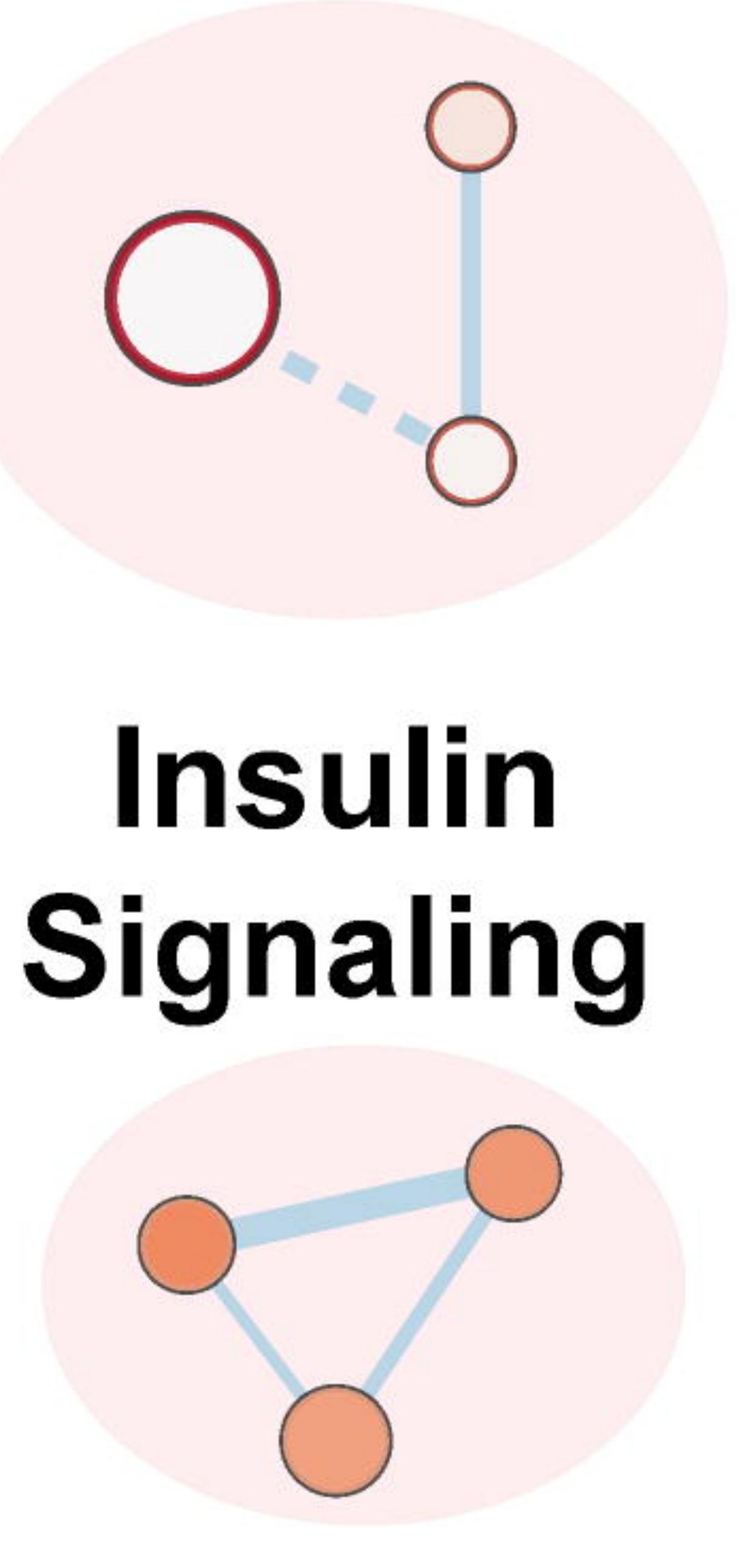


A Down-regulated in $\mathrm{Tg}$, Drug Rescued Up Toward NTg

$\begin{array}{ccc}\begin{array}{c}\text { Aerobic } \\ \text { Respiration }\end{array} & \text { LTP } & \begin{array}{c}\text { Muscle } \\ \text { Contraction }\end{array}\end{array}$

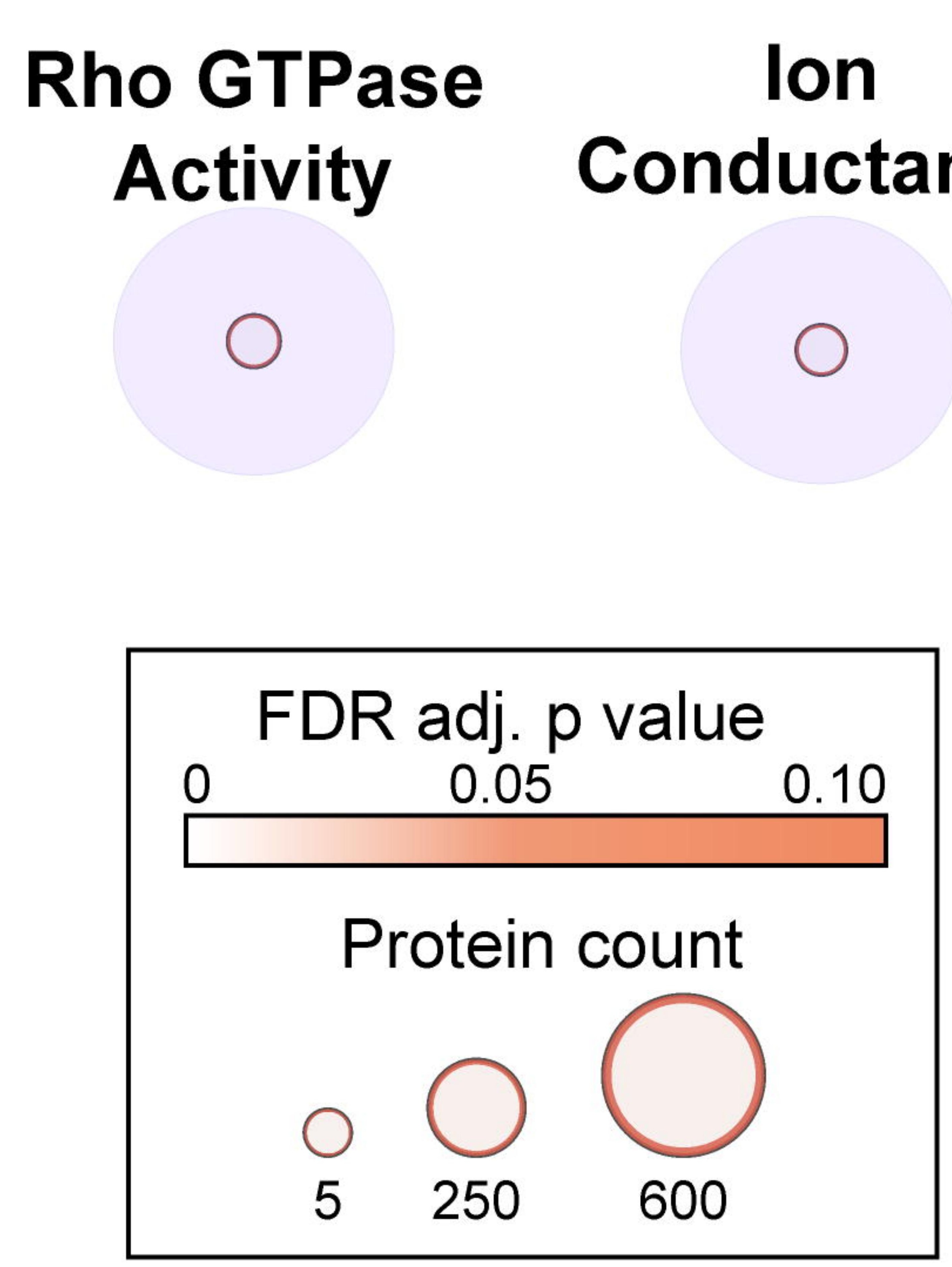

C

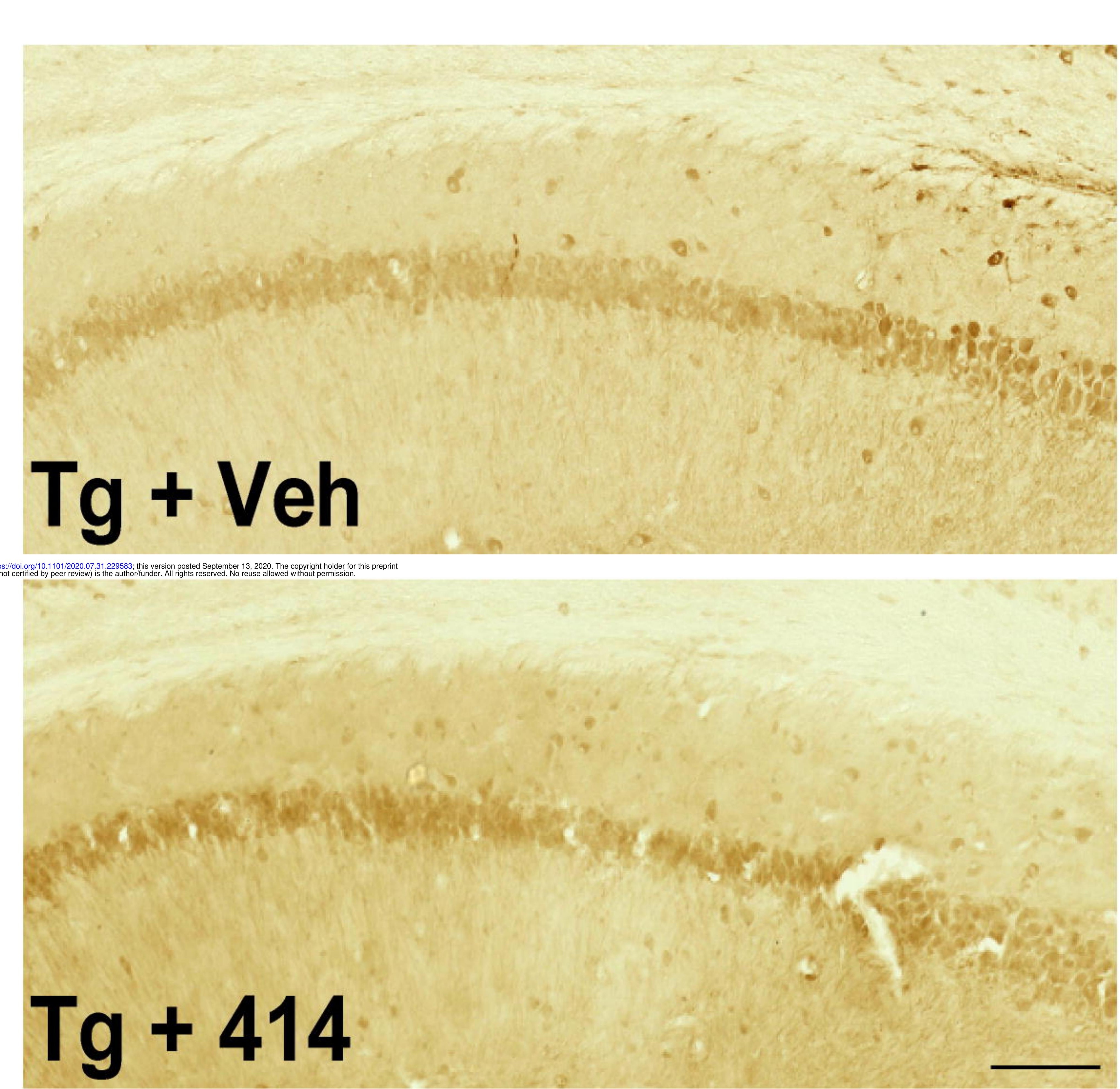

B Up-regulated in Tg, Drug Rescued Down Toward NTg

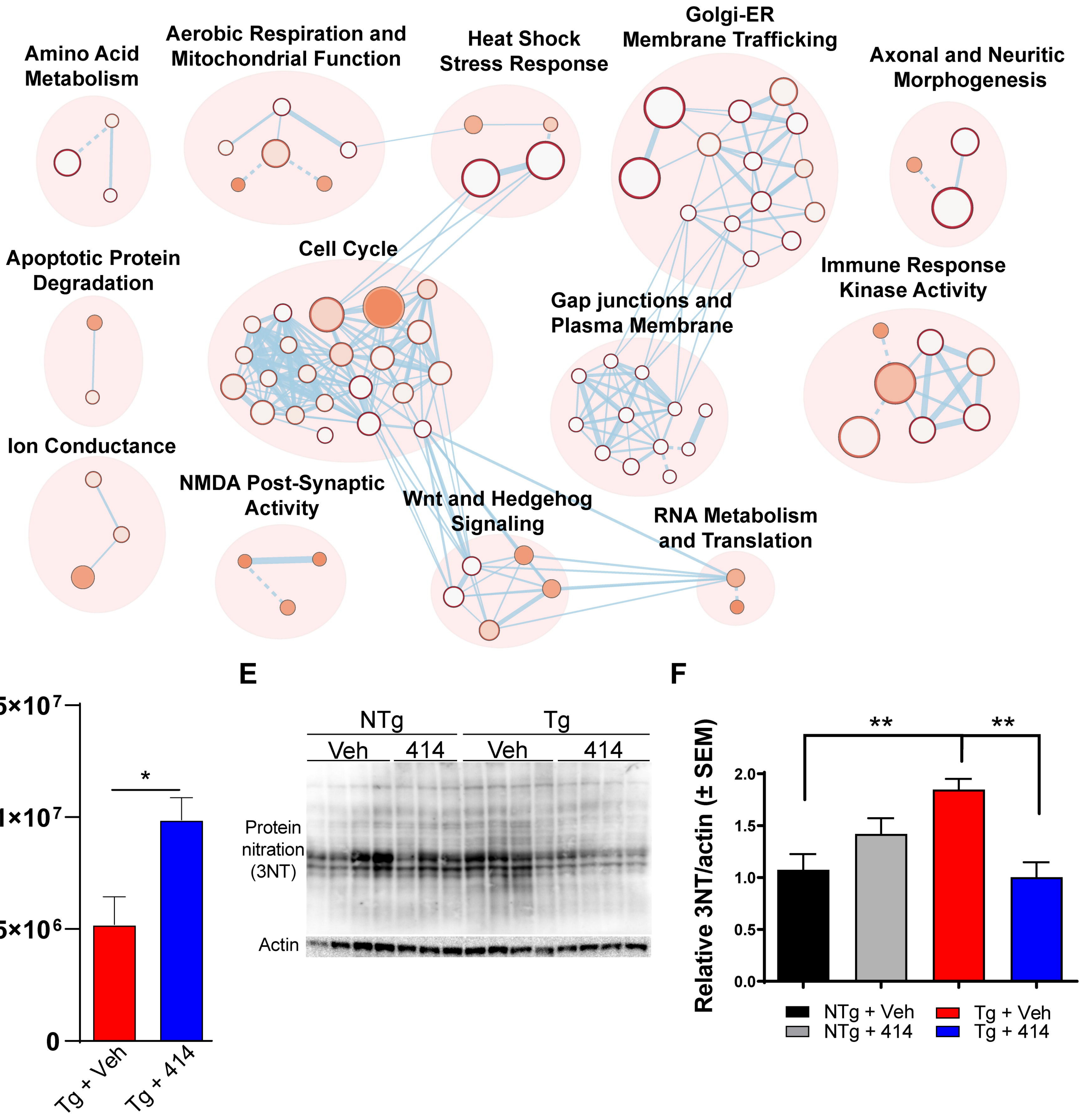


A

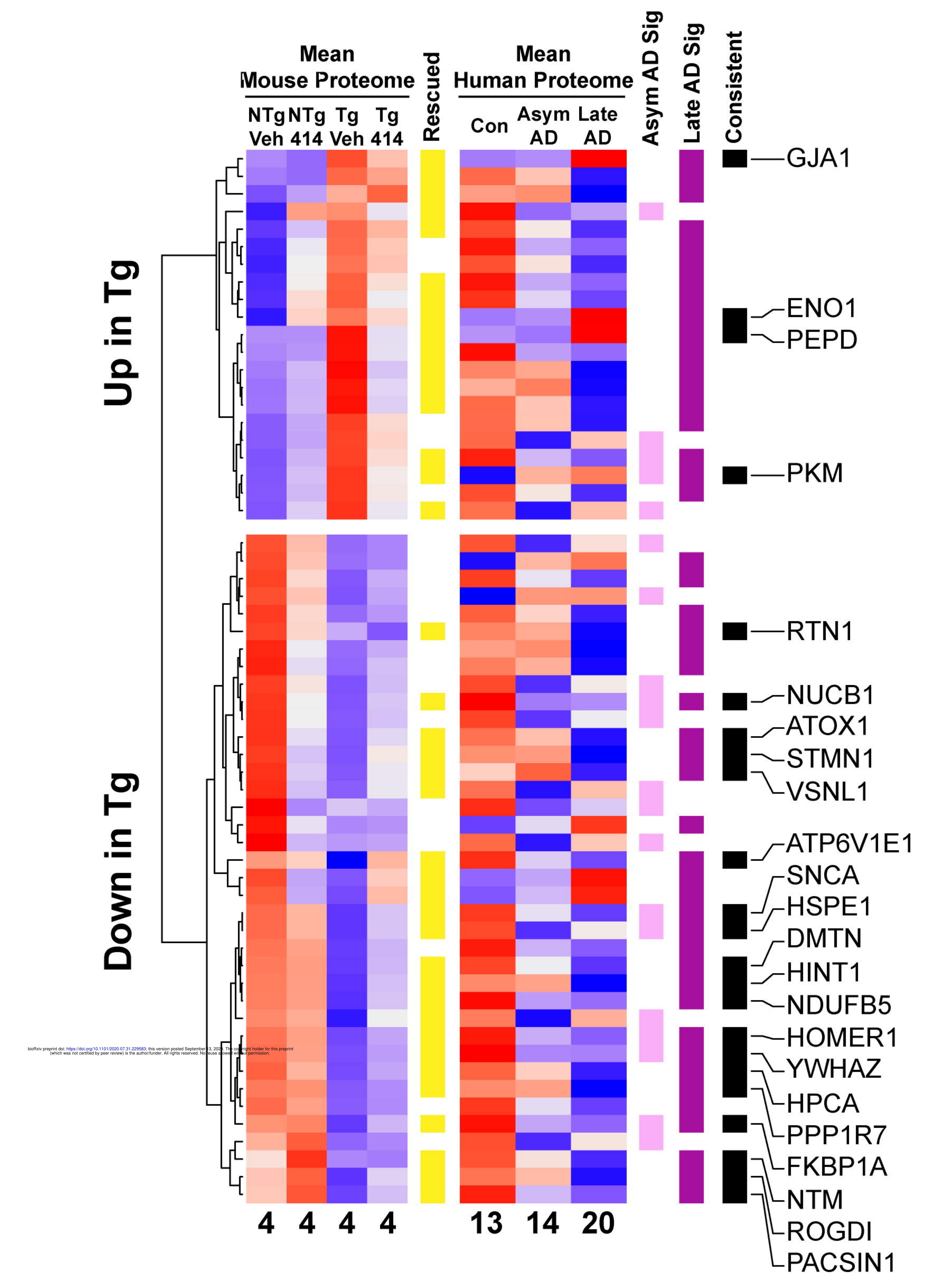

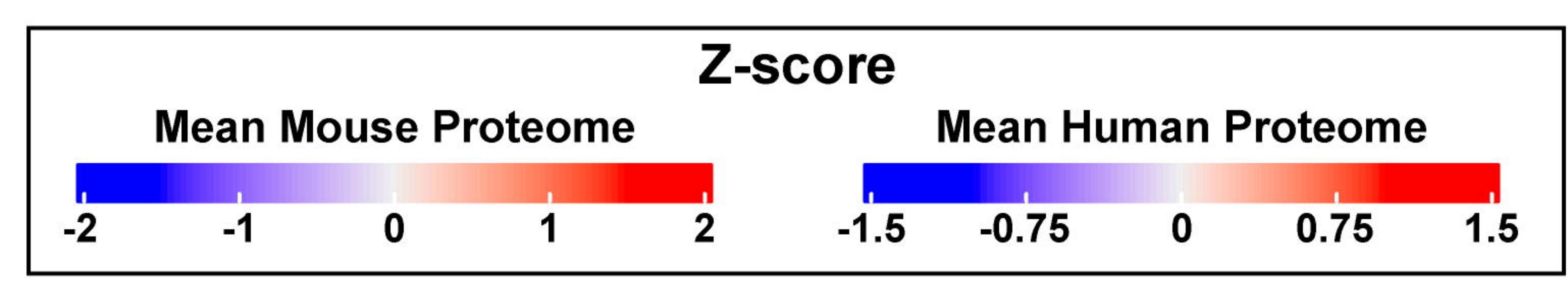

B

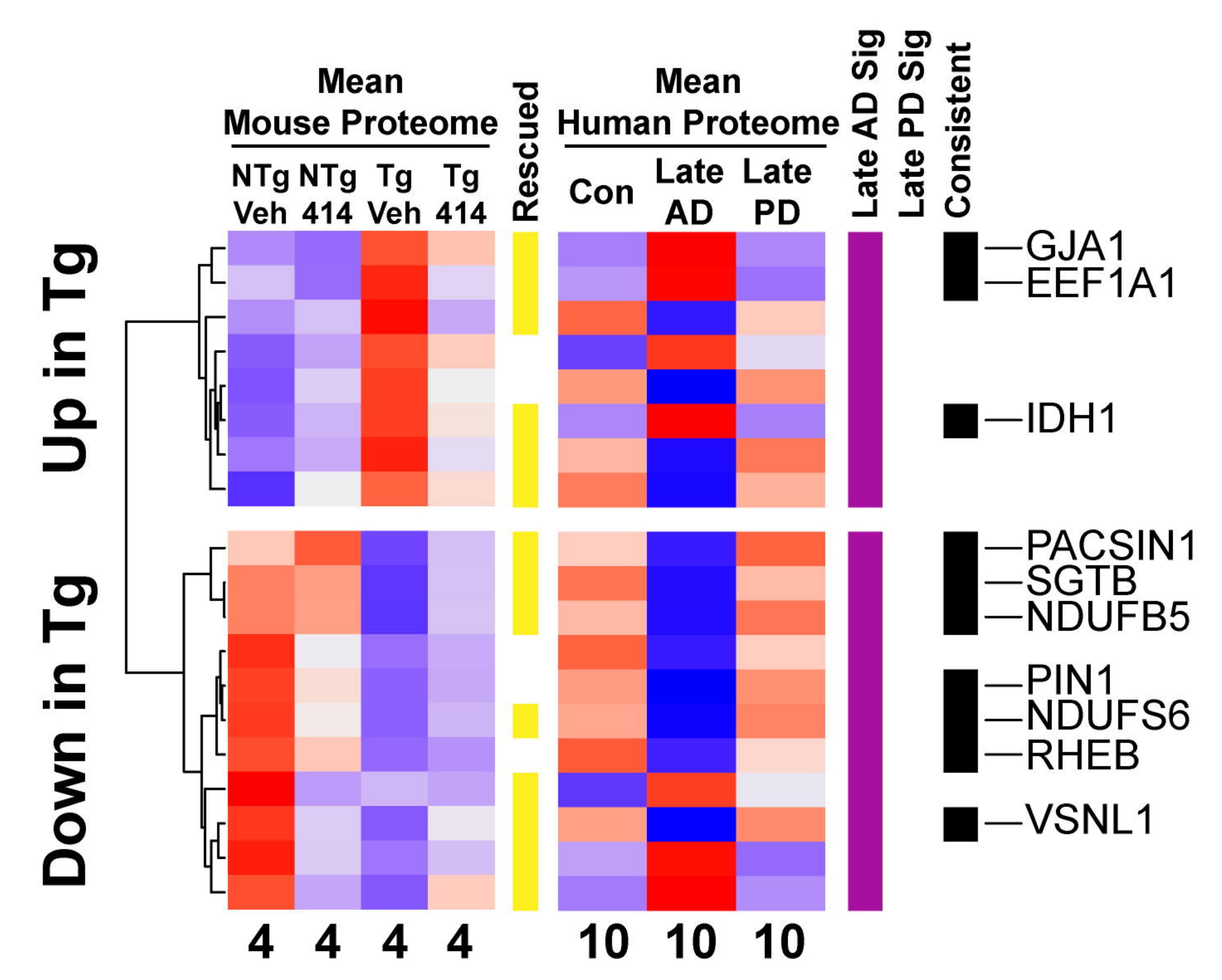

C

Seyfried, et al. 2016

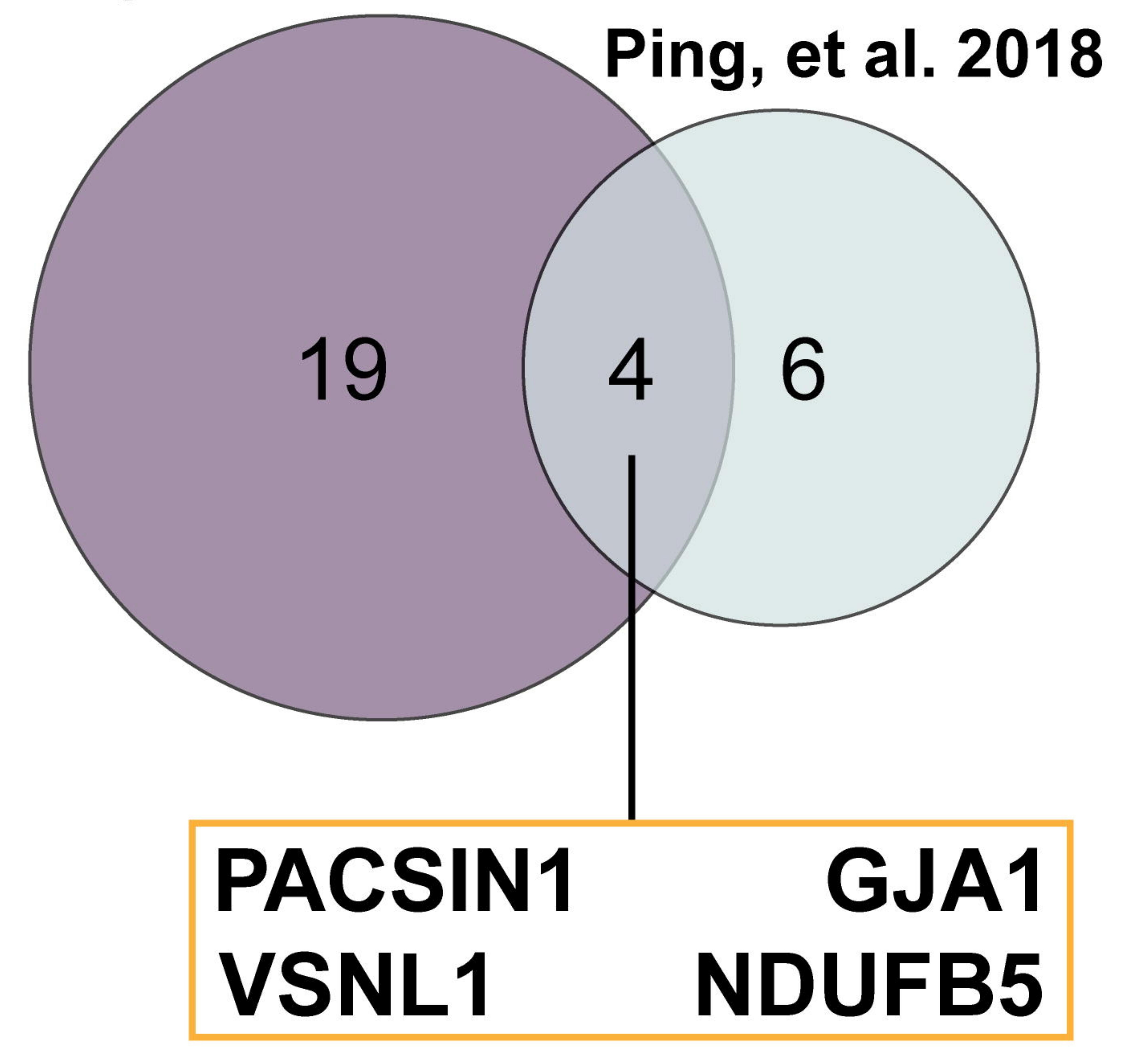

